\title{
Fully coherent energy loss effects on light hadron production in $\mathrm{pA}$ collisions
}

\author{
François Arleo, ${ }^{a}$ Florian Cougoulic ${ }^{b}$ and Stéphane Peignéc \\ ${ }^{a}$ Laboratoire Leprince-Ringuet, École polytechnique, Institut polytechnique de Paris, CNRS/IN2P3, \\ Route de Saclay, Palaiseau 91128, France \\ ${ }^{b}$ Department of Physics, The Ohio State University, \\ 191 W Woodruff Ave, Columbus, OH 43210, U.S.A. \\ ${ }^{c}$ SUBATECH UMR 6457 (IMT Atlantique, Université de Nantes, IN2P3/CNRS), \\ 4 rue Alfred Kastler, Nantes 44307, France \\ E-mail: francois.arleo@cern.ch, cougoulic.1@osu.edu, \\ peigne@subatech.in2p3.fr
}

ABSTRACT: We single out the role of fully coherent induced gluon radiation on light hadron production in $\mathrm{pA}$ collisions. The effect has the same general features as for quarkonium production, however with a richer color structure as the induced radiation depends on the global color charge of the partonic subprocess final state. Baseline predictions for light hadron nuclear suppression in $\mathrm{pPb}$ collisions at the LHC are provided, taking into account only the effect of fully coherent energy loss, which proves to be of the same order of magnitude as gluon shadowing or saturation. This underlines the need to include fully coherent energy loss in phenomenological studies of hadron production in pA collisions.

KeYwords: QCD Phenomenology

ARXIV EPRINT: 2003.06337 


\section{Contents}

1 Introduction $\quad 1$

2 Model for quarkonium nuclear suppression: a brief reminder 4

3 FCEL in light hadron nuclear suppression $\quad 6$

$\begin{array}{ll}3.1 \text { Setup and assumptions } & 6\end{array}$

$\begin{array}{lll}3.2 & \text { Implementing fully coherent energy loss } & 7\end{array}$

$\begin{array}{lll}3.3 & \text { Nuclear modification factor } R_{\mathrm{pA}} & 10\end{array}$

4 FCEL baseline predictions $\quad 11$

$\begin{array}{lll}4.1 & \text { Partonic subprocess } & 11\end{array}$

$\begin{array}{lll}4.2 & \text { Parameters and theoretical uncertainties } & 12\end{array}$

$\begin{array}{lll}4.3 & \text { Results in } \mathrm{pPb} \text { collisions at the LHC } & 14\end{array}$

$\begin{array}{ll}4.4 \text { Comparison to data } & 16\end{array}$

5 Other partonic subprocesses $\quad \mathbf{1 8}$

$\begin{array}{lll}5.1 \quad q g \rightarrow q g & 18\end{array}$

$\begin{array}{lll}5.2 g q \rightarrow q g & 18\end{array}$

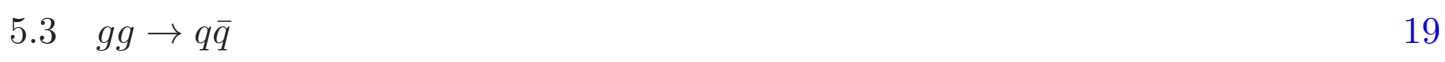

6 Discussion and outlook $\quad 21$

A Induced coherent radiation spectrum and quenching weight 22

B Color state probabilities $\rho_{\mathrm{R}}(\xi) \quad 23$

B.1 $g g \rightarrow g g \quad 24$

B.2 $q g \rightarrow q g \quad 25$

$\begin{array}{ll}\text { B.3 } g g \rightarrow q \bar{q} & 27\end{array}$

$\begin{array}{ll}\text { C Parametrization of light hadron cross section in pp collisions } & 27\end{array}$

\section{Introduction}

The wealth of hadron production data in proton-nucleus (pA) collisions at collider (RHIC, LHC) energies allows for a detailed study of parton dynamics in cold nuclear matter and of the various nuclear effects expected to occur in $\mathrm{pA}$ when compared to proton-proton (pp) collisions. Several formalisms are currently used in phenomenological studies of hadron production in high-energy pA collisions. 
In the collinear factorization approach [1], hadron production cross sections in pA collisions are evaluated assuming leading-twist QCD factorization and using the nuclear parton distribution functions (nPDFs) of the target nucleus. Collinear factorization is best justified for hadron production at large enough $p_{\perp}$, where higher-twist contributions can be neglected. The leading-twist nPDFs are obtained from global fits based on DGLAP evolution [2-5]. They exhibit gluon shadowing, namely a depletion at small $x_{2} \lesssim 10^{-2}$ of the gluon PDF in the nucleus with respect to that in a proton (see ref. [6] for a review). Gluon shadowing leads to a corresponding depletion of hadron production in $\mathrm{pA}$ with respect to pp collisions, either at RHIC (at forward rapidity) or at LHC [7, 8]. Note, however, that each $\mathrm{nPDF}$ set comes as a collection of predictions (depending on the number of parameters used in each global fit) leading to a large theoretical uncertainty on the quantitative role of shadowing, as a direct consequence of the relative lack of small- $x_{2}$ data currently included in the global fit analyses.

In the saturation formalism (see [9] for a review), hadron production cross sections in pA collisions depend on the target unintegrated (i.e., $k_{\perp}$-dependent) gluon distribution (UGD) [10]. ${ }^{1}$ This approach is in principle suitable for describing hadron production at small and moderate transverse momentum, $p_{\perp} \sim \mathcal{O}\left(Q_{s}\right)$, with $Q_{s}$ the saturation scale in the target nucleus. The UGDs are related to the quark and gluon dipole scattering amplitudes. Starting from some initial conditions at $x_{0}=10^{-2}$ incorporating the (classical) effect of nuclear $p_{\perp}$-broadening, ${ }^{2}$ the dipole amplitudes are determined at lower $x$ from the JIMWLK equations [16-21] encoding small- $x$ quantum evolution. Although the saturation formalism allows one in principle to predict the $x$ and $k_{\perp}$ dependence of UGDs, it is fair to say that the latter still contain some theoretical uncertainty, arising from the choice of initial conditions and from the approximations used to solve the small- $x$ evolution equations. While first calculations predicted a strong hadron suppression at the LHC [22], later results pointing to lesser suppression proved to be in agreement, within the theoretical and experimental uncertainties, with LHC pPb measurements [10, 23-25].

In addition to these two approaches which aim at describing the gluon distributions in large nuclei, various models have included nuclear effects like $p_{\perp}$-broadening [26] or initial-state parton energy loss in the nuclear medium $[27,28]$, in order to compute hadron production in pA collisions at RHIC and LHC. To our knowledge, however, up to now no approach has addressed the role of fully coherent energy loss (FCEL) in cold nuclear matter discussed throughout this paper. FCEL is expected in all processes where the underlying partonic process consists in forward scattering (when viewed in the target nucleus rest frame) of an incoming high-energy parton to an outgoing color charge $[29,30]$ or colourful system of partons [31]. It arises from the induced radiation of gluons with formation time

\footnotetext{
${ }^{1}$ Depending on the value of $x_{1}$ at which the projectile is probed, the partonic content of the projectile is described either by a UGD (at small enough $x_{1}$ ), or by the standard PDF of collinear factorization (at large enough $x_{1}$ ) in the so-called hybrid formalism [11, 12].

${ }^{2}$ This holds independently of the precise choice for the initial conditions, and stresses that the saturation formalism incorporates additional effects when compared to the leading-twist gluon shadowing of collinear factorization. Note that the initial conditions for the dipole scattering amplitudes may be modelled (as in the MV model $[13,14]$ ) or determined from a global analysis (using a few model assumptions) of electronproton collisions at HERA [15].
} 
$t_{\mathrm{f}}$ much larger than the medium length, $t_{\mathrm{f}} \gg L$. In this regime, the average energy loss becomes proportional to the incoming parton energy $E, \Delta E_{\mathrm{FCEL}} \propto E$ [29], thus overwhelming the parton energy loss in the Landau-Pomeranchuk-Migdal regime, $\Delta E_{\mathrm{LPM}} \propto L^{2}[32-35]$. FCEL is predicted from first principles in various formalisms [29-31, 36-39] (including the saturation formalism $[38,39]^{3}$ ) and has been shown to be a key effect to understand quarkonium suppression in pA collisions [40-42]. It is thus natural to investigate the effect of FCEL in other processes, such as open heavy flavour, light hadron, or jet production in pA collisions. In the present study, a fully detailed version of ref. [43], we focus on light hadron single inclusive production.

Our study has several motivations:

- A primary goal is to set a baseline for the quantitative role of FCEL in light hadron nuclear suppression, by taking into account only this effect. We show that similarly to quarkonium, light hadron production in pA collisions is strongly affected by FCEL, yet with novel features that will be underlined. In particular, FCEL depends on the global color charge of the parton pair produced in the partonic subprocess. This is a richer situation compared to quarkonium production at low $p_{\perp}$ [40-42], where only a color octet heavy-quark pair is produced in the subprocess. As an interesting consequence, the nuclear suppression of single hadron production is sensitive to the color states of a parton pair, and thus to unusual color factors.

- It has been suggested to use present and future data on hadron production $\left(h^{ \pm}[7,8]\right.$, $D / B$ mesons $[44,45]$, quarkonia [44]) in pA collisions as a reliable probe of $\mathrm{nPDFs}$ (and of saturation [10]), assuming other physical effects to be negligible. Our study shows that the latter assumption should be reconsidered, due to the presence of sizeable FCEL effects. In particular, FCEL should be systematically included in $\mathrm{nPDF}$ global fit analyses that use hadron production pA data. In pA collisions, electroweak processes where FCEL is absent [29] should be preferred for a direct extraction of nPDFs, as for instance weak boson [46] and Drell-Yan [47] production. It should also be reminded that no FCEL is expected in deep inelastic scattering (except in the limit of resolved photoproduction [29]), making a future electron-ion collider an ideal probe of nPDF and saturation effects only [48, 49].

- Isolating the FCEL effect is also interesting because this effect is associated, as we will see, with a quite small theoretical uncertainty. This results from FCEL being a medium-induced effect (depending on the difference between coherent radiation spectra in pA and pp collisions) fully determined within perturbative QCD.

The outline of the paper is as follows. In section 2 we remind the basics of the FCEL model for quarkonium production, which is generalized to the case of light hadron production in section 3. Baseline calculations of FCEL effects on light hadron nuclear suppression, assuming the $g g \rightarrow g g$ partonic subprocess (which should be dominant at mid-rapidity at

\footnotetext{
${ }^{3}$ Let us stress that FCEL and saturation are different effects, e.g. FCEL plays a crucial role not only at collider but also at fixed-target energies, where $x_{2}$ is not small and saturation effects are absent or negligible.
} 
LHC), are discussed and compared to experimental data in section 4. Calculations are generalized in section 5 to $q g \rightarrow q g$ and $g g \rightarrow q \bar{q}$ scattering processes. We draw conclusions and mention future studies in section 6 .

\section{Model for quarkonium nuclear suppression: a brief reminder}

The effect of fully coherent energy loss on quarkonium suppression in pA collisions was studied previously in refs. [40-42]. (The effect was also extrapolated to heavy-ion collisions in [50], in order to get a baseline for cold nuclear matter effects in those collisions.) For quarkonium production at moderate $p_{\perp}$ compared to the mass $M$ of the heavy $Q \bar{Q}$ pair, $p_{\perp} \lesssim M$, and assuming the $Q \bar{Q}$ pair to be produced in a color octet state, the partonic subprocess is similar to $g g \rightarrow G$, with a final 'massive gluon' $G$. The expression of the induced coherent radiation spectrum $\mathrm{d} I / \mathrm{d} \omega$ (with $\omega$ the soft radiated gluon energy) associated to $2 \rightarrow 1$ scattering $[29,30,39,41]$ is recalled in appendix A, see (A.1)-(A.2). ${ }^{4}$ A crucial feature of FCEL is that the energy spectrum $\omega \mathrm{d} I / \mathrm{d} \omega$ scales in $\omega / E$, leading to an average energy loss proportional to the energy $E$ of the radiating color charge [29], and thus to sizeable effects in quarkonium and more generally in hadron production in pA collisions.

In order to account for FCEL, the quarkonium differential production cross sections in pp and pA collisions are related by a shift $\varepsilon$ in the quarkonium energy [40-42],

$$
\frac{1}{A} \frac{\mathrm{d} \sigma_{\mathrm{pA}}^{\psi}}{\mathrm{d} E}(E, \sqrt{s})=\int_{0}^{\varepsilon_{\max }} \mathrm{d} \varepsilon \mathcal{P}(\varepsilon, E) \frac{\mathrm{d} \sigma_{\mathrm{pp}}^{\psi}}{\mathrm{d} E}(E+\varepsilon, \sqrt{s})
$$

where $\mathcal{P}(\varepsilon, E)$ is the energy loss probability distribution or quenching weight, with the energy loss $\varepsilon$ and quarkonium energy $E$ defined in the nucleus rest frame. The upper bound on $\varepsilon$ is $\varepsilon_{\max }=\min \left(E_{\mathrm{p}}-E, E\right),{ }^{5}$ where $E_{\mathrm{p}} \simeq s /\left(2 m_{\mathrm{p}}\right)$ is the projectile proton energy in this frame, with $m_{\mathrm{p}}$ the proton mass and $\sqrt{s}$ the proton-nucleon collision energy.

The quenching weight is related to the spectrum $\mathrm{d} I / \mathrm{d} \omega$ as [41]

$$
\mathcal{P}(\varepsilon, E)=\frac{\mathrm{d} I}{\mathrm{~d} \varepsilon} \exp \left\{-\int_{\varepsilon}^{\infty} \mathrm{d} \omega \frac{\mathrm{d} I}{\mathrm{~d} \omega}\right\}=\frac{\partial}{\partial \varepsilon} \exp \left\{-\int_{\varepsilon}^{\infty} \mathrm{d} \omega \frac{\mathrm{d} I}{\mathrm{~d} \omega}\right\} \equiv \frac{1}{E} \hat{\mathcal{P}}\left(\frac{\varepsilon}{E}\right)
$$

where $\hat{\mathcal{P}}$ is a scaling function of $x \equiv \varepsilon / E$ (a direct consequence of the scaling of $\omega \mathrm{d} I / \mathrm{d} \omega$ in $\omega / E)$. It also depends on the quarkonium transverse mass $M_{\perp} \equiv\left(M^{2}+p_{\perp}^{2}\right)^{\frac{1}{2}}$, and on the nuclear transverse momentum broadening $\ell_{\perp \mathrm{A}}$ defined by (A.5)-(A.7). The explicit expression of $\hat{\mathcal{P}}$ used in quarkonium production is obtained by setting $F_{c}=N_{c}$ in (A.4).

In view of our discussion in section 3 , let us rewrite (2.1) in two alternative ways. First, due to the scaling of $\hat{\mathcal{P}}$ in $x$, the 'energy shift' (2.1) can be naturally expressed as a rescaling of the quarkonium energy by introducing the variable

$$
z^{\prime} \equiv \frac{E}{E+\varepsilon}=\frac{1}{1+x} .
$$

\footnotetext{
${ }^{4}$ The spectrum associated to $g g \rightarrow G$ scattering is obtained from (A.1) by setting $F_{c}=N_{c}$.

${ }^{5}$ The condition $\varepsilon \leq E_{\mathrm{p}}-E$ follows from energy conservation, and $\varepsilon \leq E$ is imposed for consistency with the soft radiation approximation.
} 
Changing variable from $\varepsilon$ to $z^{\prime}$ in (2.1) we obtain (the dependence on $\sqrt{s}$ being implicit in the following)

$$
\frac{1}{A} \frac{\mathrm{d} \sigma_{\mathrm{pA}}^{\psi}(E)}{\mathrm{d} E}=\int_{z_{\min }^{\prime}}^{1} \mathrm{~d} z^{\prime} \mathcal{F}_{\text {loss }}\left(z^{\prime}\right) \frac{\mathrm{d} \sigma_{\mathrm{pp}}^{\psi}\left(E / z^{\prime}\right)}{\mathrm{d} E},
$$

where $z_{\text {min }}^{\prime}=\max \left(E / E_{\mathrm{p}}, 1 / 2\right)$ and the rescaling probability distribution $\mathcal{F}_{\text {loss }}\left(z^{\prime}\right)$ reads

$$
\mathcal{F}_{\text {loss }}\left(z^{\prime}\right)=\frac{1}{z^{\prime 2}} \hat{\mathcal{P}}\left(\frac{1-z^{\prime}}{z^{\prime}}\right)
$$

Second, in (2.1) we can trade the quarkonium energy $E$ for the quarkonium rapidity

$$
y \equiv \frac{1}{2} \ln \frac{E+p^{z}}{E-p^{z}}=\ln \frac{E+p^{z}}{M_{\perp}} \simeq \ln \frac{2 E}{M_{\perp}}
$$

leading to

$$
\frac{1}{A} \frac{\mathrm{d} \sigma_{\mathrm{pA}}^{\psi}(y)}{\mathrm{d} y}=\int_{0}^{x_{\max }} \mathrm{d} x \frac{\hat{\mathcal{P}}(x)}{1+x} \frac{\mathrm{d} \sigma_{\mathrm{pp}}^{\psi}(y+\ln (1+x))}{\mathrm{d} y} .
$$

The equations (2.4) and (2.7), corresponding respectively to an energy rescaling ( $E \rightarrow$ $\left.E / z^{\prime}\right)$ and a rapidity shift $(y \rightarrow y+\delta)$, are equivalent ways to implement FCEL, which will be useful when discussing light hadron production in section 3 . The rapidity shift $\delta$ is related to the rescaling variable $z^{\prime}$ and fractional energy loss $x$ as

$$
\delta=\ln \frac{1}{z^{\prime}}=\ln (1+x) .
$$

Since $\delta$ is invariant under longitudinal boosts, ${ }^{6}$ the form $(2.7)$ derived in the nucleus target rest frame can be directly transposed to the center-of-mass frame of an elementary protonnucleon collision.

The master equation (2.7) was applied to quarkonium production in pA collisions in refs. [40-42]. In those studies the pp cross section is taken as a parametrization fitting the pp data, and the pA cross section is obtained from (2.7) by implementing the theoretical prediction for the quenching weight $\hat{\mathcal{P}}$. In other words, (2.7) predicts the modification of the pp cross section, i.e., the nuclear modification factor

$$
R_{\mathrm{pA}}^{\psi}\left(y, p_{\perp}\right)=\frac{1}{A} \frac{\mathrm{d} \sigma_{\mathrm{pA}}^{\psi}}{\mathrm{d} y \mathrm{~d} p_{\perp}} / \frac{\mathrm{d} \sigma_{\mathrm{pp}}^{\psi}}{\mathrm{d} y \mathrm{~d} p_{\perp}}
$$

expected from the sole effect of fully coherent energy loss.

As shown in [40-42], FCEL alone explains $J / \psi$ nuclear suppression measured at fixed-target collision energies, $\sqrt{s} \lesssim 40 \mathrm{GeV}$ [51-55]. This is consistent with the fact that $\mathrm{nPDF} /$ saturation effects are expected to be mild/absent at such energies. At collider energies the central prediction of (2.7) [41] (with a narrow theoretical uncertainty band [56]) agrees well with the $J / \psi$ suppression measured in dAu collisions at RHIC

\footnotetext{
${ }^{6}$ This is also the case for the bound $x_{\max }=\min \left(1, \frac{E_{\mathrm{p}}}{E}-1\right)$ in $(2.7)$, which can be expressed as a rapidity difference. Using (2.6), we get $\ln \left(E_{\mathrm{p}} / E\right)=y_{\max }-y$, with $y_{\max } \equiv \ln \left(2 E_{\mathrm{p}} / M_{\perp}\right)$ the maximal quarkonium rapidity (at fixed $\left.p_{\perp}\right)$. Hence, $x_{\max }=\min \left(1, e^{y_{\max }-y}-1\right)$ in $(2.7)$.
} 
$(\sqrt{s}=200 \mathrm{GeV})[57,58]$ and $\mathrm{pPb}$ collisions at the $\operatorname{LHC}(\sqrt{s}=5.02 \mathrm{TeV})[59-61]$. Although experimental uncertainties still leave room for shadowing or saturation in $J / \psi$ suppression at collider energies, the results of the pure FCEL scenario tend to favour minimal estimates of those effects (e.g., nPDF sets with a moderate shadowing). Quite generally, for quarkonium but also for the production of any hadron species, the predictions with energy loss alone could be used to constrain the magnitude of other nuclear effects.

\section{$3 \quad$ FCEL in light hadron nuclear suppression}

The goal of this section is to single out the FCEL effect in light hadron production in pA collisions. Since the FCEL quenching weight scales in $\varepsilon / E$ (independently of the process where FCEL occurs), the general procedure to implement this effect will be the same as for quarkonium production, using expressions analogous to (2.4) or equivalently (2.7). However, in the case of light hadron production some new features emerge, and for clarity we present below the model in all its details.

\subsection{Setup and assumptions}

We consider light hadron single inclusive production at 'large' $p_{\perp} \gg \ell_{\perp \mathrm{A}}$, where $\ell_{\perp \mathrm{A}}$ is the transverse momentum broadening of a fast gluon across the target A. This scale, which characterizes the FCEL spectrum (A.1), is given explicitly by $\ell_{\perp \mathrm{A}}^{2}=\hat{q} L_{\mathrm{A}}$, with $L_{\mathrm{A}}$ the average path length and $\hat{q}$ the transport coefficient in cold nuclear matter parametrized by (A.7). In a leading-order picture, hadron production at $p_{\perp} \gg \ell_{\perp \mathrm{A}}$ arises from a $2 \rightarrow 2$ partonic process $a b \rightarrow c d$. The partons taken in the incoming proton and target nucleus are denoted by $a$ and $b$, respectively. The partons $c$ and $d$ of the final pair (nicknamed 'dijet' in the following) have transverse momenta $\boldsymbol{K}_{c} \equiv \boldsymbol{K}$ and $\boldsymbol{K}_{d} \simeq-\boldsymbol{K}$, with $K_{\perp} \equiv|\boldsymbol{K}| \gg \ell_{\perp \mathrm{A}}$, and energy fractions $\xi$ and $1-\xi$ with respect to the energy $E$ of parton $a$ in the nucleus rest frame. ${ }^{7}$ The $a b \rightarrow c d$ partonic process is followed by the fragmentation of one parton of the final pair into the tagged hadron, which thus inherits the transverse momentum $p_{\perp}=z K_{\perp}$, where $z$ is the fragmentation variable. The process is illustrated in figure 1 .

Let us emphasize that single inclusive hadron production in $\mathrm{pA}$ collisions can also proceed via $2 \rightarrow 1$ (typically, $g g \rightarrow g$ ) partonic channels, the final parton $K_{\perp}$ arising from multiple soft scatterings. However, such a process is relevant only for $K_{\perp} \lesssim \mathcal{O}\left(\ell_{\perp \mathrm{A}}\right)$. When $K_{\perp}>\mathcal{O}\left(\ell_{\perp \mathrm{A}}\right)$, the production process begins to single out a hard scattering, implying the presence of a hard recoil parton (of transverse momentum $\simeq-\boldsymbol{K}$ ), and should thus be interpreted as a $2 \rightarrow 2$ partonic process associated to a dijet final state. This fact can be inferred from the features of the transverse momentum distribution of a fast parton crossing a nuclear target, which is approximately Gaussian for $K_{\perp} \lesssim \ell_{\perp \mathrm{A}}$ and has an algebraic (Coulomb-like) behaviour when $K_{\perp}>\ell_{\perp \mathrm{A}}[32] .{ }^{8}$ It is also supported by the

\footnotetext{
${ }^{7}$ Note that in the terminology of refs. [30, 31, 38, 39], the processes $a b \rightarrow c d$ are nicknamed $a \rightarrow c d$ forward scatterings, emphasizing the energy flow of the process viewed in the nucleus rest frame (the incoming parton $b$ from the nucleus A being softish in this frame).

${ }^{8}$ See also appendix A of ref. [62].
} 


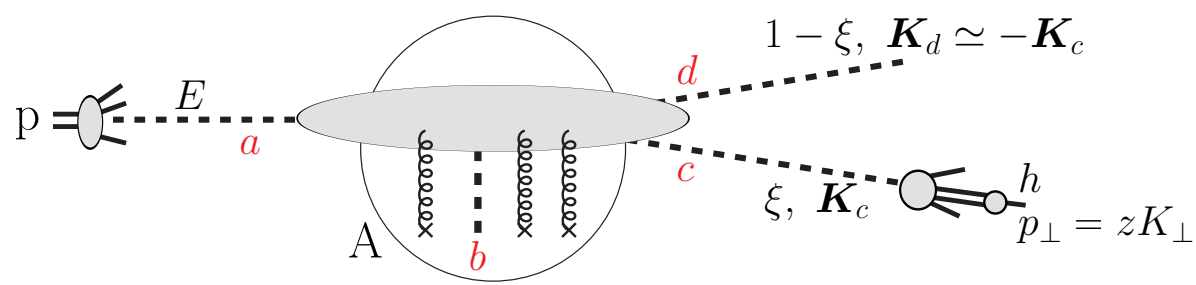

Figure 1. Contribution to light hadron production in $\mathrm{pA}$ collisions from the partonic process $a b \rightarrow c d$, as viewed in the nucleus rest frame, followed by the fragmentation $c \rightarrow h$ (represented here) or $d \rightarrow h$. In addition to the incoming parton $b$, the nucleus provides the nuclear broadening $\ell_{\perp \mathrm{A}}$ arising from parton multiple scattering.

study of refs. [63, 64], where the transition between monojet and dijet final states in pA collisions is shown to occur at quite moderate $K_{\perp} \sim \mathcal{O}\left(\ell_{\perp \mathrm{A}}\right)$.

In the target rest frame where energies are very large (transverse momenta being fixed), the parton energy fractions $\xi$ and $1-\xi$ in the dijet are equivalent to fractions of light-cone $p^{+}$-momentum (with $p^{+} \equiv p^{0}+p^{z}$ ). The latter fractions are invariant under longitudinal boosts and are related to the rapidity difference of the outgoing partons,

$$
y_{c}-y_{d}=\log \left(\frac{\xi}{1-\xi}\right)
$$

For further use we also quote the dijet invariant mass:

$$
M_{\xi}^{2}=2 K_{\perp}^{2}\left(1+\cosh \left(y_{c}-y_{d}\right)\right)=\frac{K_{\perp}^{2}}{\xi(1-\xi)} .
$$

Compared to quarkonium production, light hadron production brings two novelties: (i) the fragmentation variable $z$ between the parent parton and the tagged hadron, and (ii) the possibility to produce the dijet in different color states ( $\mathrm{SU}\left(N_{c}\right)$ irreducible representations). As we will see in the next section, the former does not bring any complication, whereas the latter makes the FCEL effect richer in light hadron production. Indeed, for a given $2 \rightarrow 2$ scattering, the associated FCEL depends on the color state of the produced parton pair [29]. This feature requires separating explicitly the different dijet color states in the hadron production cross section, and affecting each of these color states with a different induced energy loss.

\subsection{Implementing fully coherent energy loss}

As recalled in the Introduction, fully coherent radiation is associated with gluon formation times $t_{\mathrm{f}} \gg L$. In general, calculating the induced coherent radiation spectrum associated to the production of a dijet (or multi-parton system) may be complicated. There is however a simplifying limit of this problem, namely, when the induced radiation, in addition to having a formation time $t_{\mathrm{f}} \gg L$, is such that it cannot probe the dijet which thus behaves as a pointlike color charge. For this 'pointlike dijet approximation' (PDA) to hold, two conditions are a priori necessary: 
(i) At the time $t_{\mathrm{f}} \sim \omega / k_{\perp}^{2}$ of its emission, the induced radiation of energy $\omega$ and transverse momentum $k_{\perp}$ must not probe the transverse size of the parton pair. The pair having a transverse expansion velocity $\sim K_{\perp} / E$, the latter condition reads

$$
\frac{1}{k_{\perp}} \gg \frac{\omega}{k_{\perp}^{2}} \cdot \frac{K_{\perp}}{E} \Leftrightarrow \frac{\omega}{E} K_{\perp} \ll k_{\perp}
$$

Since the induced radiation $k_{\perp}$ must also be softer than the transverse 'kick' $\ell_{\perp \mathrm{A}}$ suffered by the dijet, ${ }^{9}$ we have a fortiori

$$
\frac{E \ell_{\perp \mathrm{A}}}{\omega K_{\perp}} \gg 1
$$

(ii) The induced radiation must not probe the color charges of the dijet constituents, but only see the dijet global color charge. This was shown in ref. [31] to hold within the logarithmic accuracy ${ }^{10}$

$$
\ln \left(\frac{E^{2} \ell_{\perp \mathrm{A}}^{2}}{\omega^{2} K_{\perp}^{2}}\right) \gg 1
$$

The condition (3.5) is stronger than (3.4) and thus defines the validity domain of the PDA.

The PDA brings major simplifications. First of all, in the PDA the induced spectrum $\omega \mathrm{d} I_{\mathrm{R}} / \mathrm{d} \omega$ for a dijet in color state $\mathrm{R}$ is obviously the same as for a pointlike color charge $C_{\mathrm{R}}$ of mass given by the dijet mass (3.2). This spectrum and the associated quenching weight $\hat{\mathcal{P}}_{\mathrm{R}}$ are thus obtained from (A.1)-(A.4) by replacing $M_{\perp} \rightarrow M_{\xi}$.

Similarly to the case of quarkonium production reviewed in section 2 , the quenching weight can be traded for the rescaling probability distribution $\mathcal{F}_{\mathrm{R}}\left(z^{\prime}\right)$ given by (see $(2.5)$ )

$$
\mathcal{F}_{\mathrm{R}}\left(z^{\prime}\right)=\frac{1}{z^{\prime 2}} \hat{\mathcal{P}}_{\mathrm{R}}\left(\frac{1-z^{\prime}}{z^{\prime}}\right) .
$$

Since $\mathcal{F}_{\mathrm{R}}$ depends on $\mathrm{R}$, in the present case the rescaling induced by FCEL must be done for each dijet color state separately. This leads us to express the pA cross section as an incoherent sum over the accessible dijet color states $\mathrm{R}$, and to introduce the probability $\rho_{\mathrm{R}}$ for the dijet to be in state $\mathrm{R}$. The $\rho_{\mathrm{R}}$ 's are fully determined from the $a b \rightarrow c d$ scattering amplitude (since in the PDA the induced radiation does not probe the dijet and thus conserves its color state), and turn out to depend on the energy fraction $\xi, \rho_{\mathrm{R}}=\rho_{\mathrm{R}}(\xi)$, see appendix B. ${ }^{11}$ Using $1=\sum_{\mathrm{R}} \rho_{\mathrm{R}}(\xi)$ we thus write the $\mathrm{pA}$ cross section as

$$
\frac{\mathrm{d} \sigma_{\mathrm{pA}}^{h}\left(E_{h}\right)}{\mathrm{d} E_{h}}=\sum_{\mathrm{R}} \int \mathrm{d} \xi\left[\rho_{\mathrm{R}}(\xi) \frac{\mathrm{d} \sigma_{\mathrm{pA}}^{h}\left(E_{h}, \xi\right)}{\mathrm{d} E_{h} \mathrm{~d} \xi}\right],
$$

\footnotetext{
${ }^{9}$ See the discussion in section 2.2 of ref. [31].

${ }^{10}$ It was shown in [31] that (3.5) is a sufficient condition for the induced radiation to depend only on the dijet global color state R. It is not difficult to verify from ref. [31] that this property is spoiled beyond the logarithmic accuracy (3.5), so that (3.5) is also a necessary condition.

${ }^{11}$ In addition to the energy fraction $\xi$ and color state $\mathrm{R}$, the $\rho_{\mathrm{R}}$ 's obviously depend on the specific $a b \rightarrow c d$ channel (and not only on the final dijet color state). For instance, the octet final states produced respectively in $g g \rightarrow g g$ and $g g \rightarrow q \bar{q}$ processes are associated with different probabilities $\rho_{\mathbf{8}}(\xi)$, see figure 9 .
} 
where the quantity in brackets is the pA cross section to find a hadron of energy $E_{h}$ in a dijet of color state $\mathrm{R}$ and with energy fractions $\xi$ and $1-\xi$. Note that the latter cross section is inclusive in which parton of the dijet fragments into the tagged hadron.

Another simplification of the PDA is that the energy rescaling (by a factor $z^{\prime}$ ) conserves the dijet internal structure, and in particular the energy fractions $\xi$ and $1-\xi$. The two partons thus inherit the same rescaling, and in turn the tagged hadron too, independently of the additional rescaling (by a factor $z$ ) inherent to the fragmentation process. ${ }^{12}$ As a consequence, FCEL can be implemented by replacing in (3.7), for each color state R, the quantity in brackets by the similar quantity in pp collisions (multiplied by the atomic mass number $A$ ), but with $E_{h}$ rescaled by $z^{\prime}$ with probability $\mathcal{F}_{\mathrm{R}}\left(z^{\prime}\right)$,

$$
\frac{1}{A} \frac{\mathrm{d} \sigma_{\mathrm{pA}}^{h}\left(E_{h}\right)}{\mathrm{d} E_{h}}=\sum_{\mathrm{R}} \int \mathrm{d} \xi \rho_{\mathrm{R}}(\xi) \int_{z_{\min }^{\prime}}^{1} \mathrm{~d} z^{\prime} \mathcal{F}_{\mathrm{R}}\left(z^{\prime}\right) \frac{\mathrm{d} \sigma_{\mathrm{pp}}^{h}\left(E_{h} / z^{\prime}, \xi\right)}{\mathrm{d} E_{h} \mathrm{~d} \xi} .
$$

Eq. (3.8) generalizes (2.4) used in quarkonium production to the case of light hadron production. In the PDA the energy rescaling is equivalent to applying the same rapidity shift $\delta$ to the dijet, its constituent partons, and the tagged hadron. Trading the hadron energy $E_{h}$ for its rapidity $y$ and using (3.6), the expression (3.8) becomes

$$
\frac{1}{A} \frac{\mathrm{d} \sigma_{\mathrm{pA}}^{h}\left(y, p_{\perp}\right)}{\mathrm{d} y \mathrm{~d} p_{\perp}}=\sum_{\mathrm{R}} \int \mathrm{d} \xi \rho_{\mathrm{R}}(\xi) \int_{0}^{x_{\max }} \frac{\mathrm{d} x}{1+x} \hat{\mathcal{P}}_{\mathrm{R}}\left(x, \ell_{\perp \mathrm{A}}, M_{\xi}\right) \frac{\mathrm{d} \sigma_{\mathrm{pp}}^{h}\left(y+\delta, p_{\perp}, \xi\right)}{\mathrm{d} y \mathrm{~d} p_{\perp} \mathrm{d} \xi}
$$

where $\delta=\ln (1+x), x_{\max }=\min \left(1, e^{y_{\max }-y}-1\right),{ }^{13}$ and the dependence of the pp and pA cross sections on the hadron $p_{\perp}$ is now explicit, as well as that of $\hat{\mathcal{P}}_{\mathrm{R}}$ on the broadening $\ell_{\perp \mathrm{A}}$ and dijet mass $M_{\xi}$. Note that the 'hard scale' $K_{\perp}$ to be used in the expression of $M_{\xi}$ (see (3.2)) reads $K_{\perp}=p_{\perp} / z$, to account for the rescaling of momenta in the fragmentation process. In our approach, the momentum fraction $z$ of the tagged hadron w.r.t. its parent parton is treated as a parameter, see section 4.2. Eq. (3.9) generalizes (2.7) to light hadron production, and will be used in the following to predict the effect of FCEL on the light hadron nuclear modification factor $R_{\mathrm{pA}}$. We have checked that the typical values of $x$ contributing to (3.9) satisfy $\ln \left(\ell_{\perp \mathrm{A}}^{2} / x^{2} K_{\perp}^{2}\right) \sim 3-4$ and thus fulfill the condition (3.5), justifying a posteriori the PDA and the above implementation of FCEL.

Let us note that the pp and pA cross sections appearing in (3.9) are evaluated at the same $p_{\perp}$. There are two reasons for that. First, as explained in the Introduction, our goal is to single out FCEL, and in (3.9) we thus neglect the shift in $p_{\perp}$ due to nuclear broadening in pA vs. pp. ${ }^{14}$ Second, the FCEL effect itself can in principle affect the transverse momenta of the dijet constituents, and thus induce a difference between the hadron $p_{\perp}$ in the $\mathrm{pA}$ and pp cross sections. However, this effect should be neglected in the PDA, where in addition

\footnotetext{
${ }^{12}$ This holds because FCEL and fragmentation processes occur at different time scales [41].

${ }^{13}$ This is the same as for quarkonium production (see footnote 6 ), with $y_{\max }$ being now the maximal hadron rapidity. In the proton-nucleon collision c.m. frame, $y_{\max }=\ln \left(\sqrt{s} / p_{\perp}\right)$.

${ }^{14}$ It should be noted that there is no contradiction in isolating the FCEL effect while neglecting $p_{\perp}$ broadening, despite the fact that the former is induced by the latter, as illustrated by the vanishing of $\hat{\mathcal{P}}_{\mathrm{R}}$ when the nuclear broadening $\ell_{\perp \mathrm{A}}^{2}-\ell_{\perp \mathrm{p}}^{2}$ vanishes, see (A.4). In our approach, the role of $p_{\perp}$-broadening is simply to specify the quantity $\ell_{\perp \mathrm{A}}$ to be used in the FCEL quenching weight $\hat{\mathcal{P}}_{\mathrm{R}}$.
} 
to the internal energy fraction $\xi$, the dijet invariant mass (3.2) must be conserved. As a consequence, $K_{\perp}$ and thus $p_{\perp}=z K_{\perp}$ are conserved.

To conclude this section, let us stress that our implementation of FCEL consists in a different organization of the perturbative expansion as compared to next-to-leading order (NLO) approaches evaluating absolute pA cross sections (see e.g. refs. [65-67] for single inclusive hadron and refs. [68-70] for quarkonium production). These studies in principle account for the induced radiation of a single gluon, as part of all NLO corrections. In our approach, the induced radiation is resummed to all orders through the expression (2.2) of the quenching weight. This should be meaningful when the spectrum (A.1) cannot be viewed as a genuine NLO correction, i.e., when $\alpha_{s} \ln \left(E^{2} \ell_{\perp \mathrm{A}}^{2} / \omega^{2} K_{\perp}^{2}\right) \gtrsim \mathcal{O}(1)$, consistently with the logarithmic accuracy (3.5) used in our study.

\subsection{Nuclear modification factor $\boldsymbol{R}_{\mathrm{pA}}$}

The nuclear suppression of a light hadron $h$, in minimum bias pA collisions as compared to pp collisions, is commonly represented by the ratio

$$
R_{\mathrm{pA}}^{h}\left(y, p_{\perp}\right)=\frac{1}{A} \frac{\mathrm{d} \sigma_{\mathrm{pA}}^{h}}{\mathrm{~d} y \mathrm{~d} p_{\perp}} / \frac{\mathrm{d} \sigma_{\mathrm{pp}}^{h}}{\mathrm{~d} y \mathrm{~d} p_{\perp}}
$$

where $y$ is the hadron rapidity in the c.m. frame of an elementary proton-nucleon collision.

Using (3.9) we obtain

$$
R_{\mathrm{pA}}^{h}\left(y, p_{\perp}\right)=\int_{0}^{x_{\max }} \frac{\mathrm{d} x}{1+x}\left\langle\sum_{\mathrm{R}} \rho_{\mathrm{R}}(\xi) \hat{\mathcal{P}}_{\mathrm{R}}\left(x, \ell_{\perp \mathrm{A}}, M_{\xi}\right)\right\rangle_{y+\delta, p_{\perp}} \frac{\frac{\mathrm{d} \sigma_{\mathrm{pp}}^{h}\left(y+\delta, p_{\perp}\right)}{\mathrm{d} y \mathrm{~d} p_{\perp}}}{\frac{\mathrm{d} \sigma_{\mathrm{pp}}^{h}\left(y, p_{\perp}\right)}{\mathrm{d} y \mathrm{~d} p_{\perp}}}
$$

where \langle\rangle$_{y, p_{\perp}}$ denotes the $\xi$-average in pp dijet events where a hadron of rapidity $y$ and transverse momentum $p_{\perp}$ is produced, namely,

$$
\langle f(\xi)\rangle_{y, p_{\perp}} \equiv \int \mathrm{d} \xi f(\xi) g_{y, p_{\perp}}(\xi) ; \quad g_{y, p_{\perp}}(\xi)=\frac{\frac{\mathrm{d} \sigma_{\mathrm{pp}}^{h}\left(y, p_{\perp}, \xi\right)}{\mathrm{d} y \mathrm{~d} p_{\perp} \mathrm{d} \xi}}{\int \mathrm{d} \xi \frac{\mathrm{d} \sigma_{\mathrm{pp}}^{h}\left(y, p_{\perp}, \xi\right)}{\mathrm{d} y \mathrm{~d} p_{\perp} \mathrm{d} \xi}} .
$$

The functions $\hat{\mathcal{P}}_{\mathrm{R}}$ and $\rho_{\mathrm{R}}$ being defined in appendices $\mathrm{A}$ and $\mathrm{B}$, the factor $\left\langle\sum_{\mathrm{R}} \rho_{\mathrm{R}} \hat{\mathcal{P}}_{\mathrm{R}}\right\rangle$ in (3.11) could in principle be evaluated knowing the triple $\left(y, p_{\perp}, \xi\right)$ differential pp cross section, e.g. within collinear factorization once a given set of PDFs and fragmentation functions is chosen. In order to minimize the number of model assumptions, we will instead use the following procedure.

From the (generalized) mean value theorem, given some $\xi$-average \langle\rangle defined using a probability density $g(\xi)$, for any continuous function $f(\xi)$ there is a value $\bar{\xi}$ (belonging to the support of $g(\xi))$ such that $\langle f(\xi)\rangle=f(\bar{\xi})$. The nuclear modification factor (3.11) can thus be written as

$$
\begin{aligned}
& R_{\mathrm{pA}}^{h}\left(y, p_{\perp}, \bar{\xi}\right)=\sum_{\mathrm{R}} \rho_{\mathrm{R}}(\bar{\xi}) R_{\mathrm{pA}}^{\mathrm{R}}\left(y, p_{\perp}, \bar{\xi}\right), \\
& R_{\mathrm{pA}}^{\mathrm{R}}\left(y, p_{\perp}, \bar{\xi}\right)=\int_{0}^{x_{\mathrm{max}}} \frac{\mathrm{d} x}{1+x} \hat{\mathcal{P}}_{\mathrm{R}}\left(x, \ell_{\perp \mathrm{A}}, M_{\bar{\xi}}\right) \frac{\frac{\mathrm{d} \sigma_{\mathrm{pp}}^{h}\left(y+\delta, p_{\perp}\right)}{\mathrm{d} y \mathrm{~d} p_{\perp}}}{\frac{\mathrm{d} \sigma_{\mathrm{pp}}^{h}\left(y, p_{\perp}\right)}{\mathrm{d} y \mathrm{~d} p_{\perp}}} .
\end{aligned}
$$


Thus, $R_{\mathrm{pA}}^{h}$ is the color average (over the accessible dijet color states $\mathrm{R}$ ) of the modification factors $R_{\mathrm{pA}}^{\mathrm{R}}$, corresponding to a hadron produced from a dijet in state $\mathrm{R}$. The uncertainty associated to the choice of the parameter $\bar{\xi}$ will be estimated by varying $\bar{\xi}$ in the support interval of $g_{y, p_{\perp}}(\xi)$ defined in (3.12). The latter will be chosen as the interval $[0.25,0.75]$, motivated by experimental measurements as discussed in section 4.2. The above procedure avoids the calculation of $\left\langle\sum_{R} \rho_{R} \hat{\mathcal{P}}_{R}\right\rangle$ appearing in (3.11) at relatively low cost since the uncertainty band associated to the variation of $\bar{\xi}$ turns out to be quite narrow (see section 4 and figure 4).

We conclude this section by noting that in some special cases of partonic processes, the FCEL spectrum is negative (namely, when $F_{c}<0$, see (A.1)-(A.2)), corresponding to an induced energy gain in pA relative to pp collisions. Then (3.14) should be replaced by

$$
\left.R_{\mathrm{pA}}^{\mathrm{R}}\left(y, p_{\perp}, \bar{\xi}\right)\right|_{\text {gain }}=\int_{0}^{x_{\max }} \mathrm{d} x(1+x) \hat{\mathcal{P}}_{\mathrm{R}}\left(x, \ell_{\perp \mathrm{A}}, M_{\bar{\xi}}\right) \frac{\frac{\mathrm{d} \sigma_{\mathrm{pp}}^{h}\left(y-\delta, p_{\perp}\right)}{\mathrm{d} y \mathrm{~d} p_{\perp}}}{\frac{\mathrm{d} \sigma_{\mathrm{pp}}^{h}\left(y, p_{\perp}\right)}{\mathrm{d} y \mathrm{~d} p_{\perp}}},
$$

where $x_{\max }=\min \left(1, e^{y_{\max }+y}-1\right)$, and $\hat{\mathcal{P}}_{\mathrm{R}}$ is associated to the opposite of the radiation spectrum (A.1) and understood as an energy gain probability density. Eq. (3.15) is obtained as follows. In the case of energy gain, the sign of the energy shift (see (2.1)) is changed, $E+\varepsilon \rightarrow E-\varepsilon$, the rescaling variable $\frac{1}{z^{\prime}}=\frac{E+\varepsilon}{E}=1+x($ see $(2.3))$ becomes $\frac{1}{z^{\prime}}=1-x \simeq \frac{1}{1+x}$, and the rapidity $\operatorname{shift} \delta=\ln \frac{1}{z^{\prime}}=\ln (1+x)$ in (3.15) thus comes with a minus sign.

Eq. (3.15) applies for instance to the case of the $q g \rightarrow q g$ partonic process (discussed in section 5.1) when the final $q g$ pair is color triplet, $\mathrm{R}=\mathbf{3}$. Indeed, the color factor (A.2) then reads $F_{c}=2 C_{F}-N_{c}=-1 / N_{c}<0$. A physical interpretation of fully coherent energy gain for $q g \rightarrow[q g]_{3}$ scattering can be found in ref. [30].

\section{FCEL baseline predictions}

We now evaluate light hadron nuclear suppression due to the sole FCEL effect, based on eqs. (3.13)-(3.14). In the present section, we assume the $g g \rightarrow g g$ channel to be dominant, which is a reasonable assumption at the LHC, as recalled in section 4.1. The procedure to compute $R_{\mathrm{pA}}^{\mathrm{R}}\left(y, p_{\perp}\right)$ (for each dijet color state $\mathrm{R}$ ) and the hadron nuclear modification factor $R_{\mathrm{pA}}^{h}\left(y, p_{\perp}\right)$, as well as their associated uncertainties, is presented in section 4.2. Results in $\mathrm{pPb}$ collisions at the $\mathrm{LHC}$ will be shown in section 4.3 , and compared to available experimental data in section 4.4.

The phenomenology of hadron production in dAu collisions at RHIC energies, for which FCEL effects are also expected, is left for future work.

\subsection{Partonic subprocess}

Eqs. (3.13)-(3.14) allow for computing the hadron nuclear modification factor $R_{\mathrm{pA}}^{h}\left(y, p_{\perp}\right)$ for any given underlying $2 \rightarrow 2$ partonic process. The observable modification factor could be then obtained by averaging the latter $R_{\mathrm{pA}}^{h}$ 's, with weights given by the relative contributions of each subprocess to the inclusive hadron production cross section. Those weights could in principle be accessed through fixed-order perturbative QCD calculations, but may differ from one calculation to another depending on the choice of factorization 
scales, parton distributions and fragmentation functions. In practice, however, hadron production at the LHC is dominated by gluon-initiated processes at not too large $p_{\perp}$ $\left(p_{\perp} \lesssim 50 \mathrm{GeV}\right)$ and not too large rapidity $\left(|y| \lesssim 2\right.$ at $\left.p_{\perp}=25 \mathrm{GeV}\right)[71]$, thanks to the huge gluon flux in the projectile and target at small values of $x_{1}$ and $x_{2}$. In addition, light hadron production proceeds predominantly by gluon rather than quark fragmentation [71].

For these reasons, the hadron nuclear modification factor in pA collisions at the LHC is determined in the present section assuming the sole $g g \rightarrow g g$ partonic subprocess, which should be a solid assumption around mid-rapidity. Moreover, we expect the FCEL effect to be qualitatively similar for all partonic subprocesses. In order to illustrate this point, we will give in section 5 our predictions for the $q g \rightarrow q g, g q \rightarrow q g$ and $g g \rightarrow q \bar{q}$ channels. The $q g \rightarrow q g$ channel is interesting in itself because it becomes important, or even dominant, at $y \gtrsim 3-4$, where the projectile hadron is probed at higher momentum fraction, $x_{1} \propto e^{y}$, leading to a significant contribution of quark-induced processes. Similarly, the $g q \rightarrow q g$ channel (discussed in section 5.2) becomes important at $-y \gtrsim 3-4$, where the target nucleus is probed at higher momentum fraction, $x_{2} \propto e^{-y}$.

It will be important for phenomenology to quantify the FCEL effect in the general case where several partonic processes are of comparable importance in a given rapidity region. This is briefly discussed in section 6 and will be the subject of a future study.

\subsection{Parameters and theoretical uncertainties}

The calculation of $R_{\mathrm{pA}}^{\mathrm{R}}$ follows the procedure developed for quarkonium studies in refs. [41, $42,50]$. The goal is to determine hadron suppression with the least number of assumptions and parameters, the latter being then varied for a proper determination of theoretical uncertainties.

In order to minimize the model dependence, the double differential pp cross section $\mathrm{d} \sigma_{\mathrm{pp}}^{h} / \mathrm{d} y \mathrm{~d} p_{\perp}$ entering (3.14) is not taken from theory but determined from a fit to the data. A simple functional form using few parameters allows for an accurate description of the pp cross section, see appendix C. Since (3.14) involves a cross section ratio, only one of these parameters, namely $n$ in eq. (C.1), proves necessary for the calculation of $R_{\mathrm{pA}}^{\mathrm{R}}$. The value used here, $n=15 \pm 5$, is determined from a fit to $\mathrm{CMS} \mathrm{pPb}$ data at $\sqrt{s}=5.02 \mathrm{TeV}$ [72]. As the only theoretical input in (3.14) is the quenching weight $\hat{\mathcal{P}}_{\mathrm{R}}$, our calculation of hadron suppression expected from FCEL is directly sensitive to the induced gluon spectrum (A.1)(A.2) determined from first principles.

The average (3.13) over the gluon pair (more generally, dijet) color states has a smooth dependence on the parameter $\bar{\xi}$. The default value chosen for $\bar{\xi}$ corresponds to the symmetric configuration of two jets of equal rapidity, leading to $\bar{\xi}=0.5$, which is most likely according to dihadron correlation measurements in pp collisions at the LHC [73]. The uncertainty associated to the choice of $\bar{\xi}$ is estimated by varying $\bar{\xi}$ by half its default value, $\bar{\xi}=0.50 \pm 0.25,{ }^{15}$ which corresponds to a rapidity difference between the two back-to-back jets of approximately $\Delta y= \pm \ln (\bar{\xi} /(1-\bar{\xi})) \simeq \pm 1$ unit.

\footnotetext{
${ }^{15}$ Note that the dijet invariant mass $M_{\bar{\xi}}$ (entering the quenching weight in (3.14)) is symmetric in $\bar{\xi} \leftrightarrow 1-\bar{\xi}$, and so are the color probabilities $\rho_{\mathrm{R}}$ for the $g g \rightarrow g g$ channel considered in the present section, leading to the same symmetry for $R_{\mathrm{pA}}^{h}, R_{\mathrm{pA}}^{h}\left(y, p_{\perp}, \bar{\xi}\right)=R_{\mathrm{pA}}^{h}\left(y, p_{\perp}, 1-\bar{\xi}\right)$.
} 
The (average) momentum fraction $z$ of the fragmenting parton carried away by the measured hadron depends on the shape of fragmentation functions (and on the hadron species), which are still poorly known. Based on NLO calculations of hadron production at the LHC $[71,74]$, we use $z=0.6 \pm 0.2$ in the calculations to come.

Finally, for the transport coefficient we use $\hat{q}_{0}=0.07 \pm 0.02 \mathrm{GeV}^{2} / \mathrm{fm}$, which is consistent with phenomenological estimates from a variety of processes and reactions, for instance radiative energy loss in quarkonium production [41], Drell-Yan production [75] and hadron production in semi-inclusive DIS [76], nuclear $p_{\perp}$-broadening of Drell-Yan and $J / \psi$ from fixed-target to collider energies [77], and phenomenological extractions of the saturation scale in a proton from global fits to DIS data [15]. ${ }^{16}$

In order to estimate the theoretical uncertainties, these quantities are varied around their central value, $S^{0} \equiv\left\{n, \bar{\xi}, z, \hat{q}_{0}\right\}$. On top of the central prediction assuming $S^{0}$, predictions will be made with the sets of parameters $S_{k}^{ \pm} \equiv\left\{p_{k}^{ \pm}, p_{i \neq k}\right\}$, where the $k^{\text {th }}$ parameter is set to its minimal $\left(p_{k}^{-}\right)$or maximal $\left(p_{k}^{+}\right)$value, while the other parameters are fixed to their central values.

Assuming that the parameters are uncorrelated, the uncertainty band of our predictions is determined using the Hessian method [78] (as was also done in ref. [50]),

$$
\begin{aligned}
& \left(\Delta R_{\mathrm{pA}}^{+}\right)^{2}=\sum_{k}\left[\max \left\{R_{\mathrm{pA}}\left(S_{k}^{+}\right)-R_{\mathrm{pA}}\left(S^{0}\right), R_{\mathrm{pA}}\left(S_{k}^{-}\right)-R_{\mathrm{pA}}\left(S^{0}\right), 0\right\}\right]^{2}, \\
& \left(\Delta R_{\mathrm{pA}}^{-}\right)^{2}=\sum_{k}\left[\max \left\{R_{\mathrm{pA}}\left(S^{0}\right)-R_{\mathrm{pA}}\left(S_{k}^{+}\right), R_{\mathrm{pA}}\left(S^{0}\right)-R_{\mathrm{pA}}\left(S_{k}^{-}\right), 0\right\}\right]^{2}
\end{aligned}
$$

with $\left\{n, n^{-}, n^{+}\right\}=\{15,10,20\},\left\{\bar{\xi}, \bar{\xi}^{-}, \bar{\xi}^{+}\right\}=\{0.5,0.25,0.75\},\left\{z, z^{-}, z^{+}\right\}=\{0.6,0.4,0.8\}$, and (in unit $\mathrm{GeV}^{2} / \mathrm{fm}$ ) $\left\{\hat{q}_{0}, \hat{q}_{0}^{-}, \hat{q}_{0}^{+}\right\}=\{0.07,0.05,0.09\}$. In the next section we will display (see figure 4 ) the individual contributions $R_{\mathrm{pA}}\left(S_{k}^{ \pm}\right)$to the total theoretical uncertainty defined in (4.1).

Finally, as in ref. [41] we set $\alpha_{s}=0.5$ for the strong coupling constant, ${ }^{17} L_{\mathrm{Pb}}=10.11 \mathrm{fm}$ for the average path length in the lead nucleus (determined within Glauber theory using realistic nuclear density profiles), and $L_{\mathrm{p}}=1.5 \mathrm{fm}$ for that in a proton.

The quoted uncertainty of $R_{\mathrm{pA}}$ reflects only the error propagation of the parameters identified in our study, similarly to what is currently done in the uncertainty determination of parton distribution functions. We do not attempt to quantify the uncertainties inherent to the model assumptions, which are however few and justified in section 3 .

In particular, let us mention that we will present FCEL baseline predictions in the interval $1 \mathrm{GeV} \leq p_{\perp} \leq 10 \mathrm{GeV}$, while our approach is justified in the domain $p_{\perp}>\mathcal{O}\left(\ell_{\perp \mathrm{A}}\right)$, where $2 \rightarrow 2$ partonic processes are expected to be dominant (see section 3.1). In the range $1 \mathrm{GeV} \leq p_{\perp} \lesssim \mathcal{O}\left(\ell_{\perp \mathrm{A}}\right)$ (which, given standard values of $\ell_{\perp \mathrm{A}}$, is however quite restricted), the presence of $2 \rightarrow 1$ partonic processes may affect our results. In principle, the FCEL effect on $2 \rightarrow 1$ processes (such as $g g \rightarrow g$ ) contributing to light hadron production at $p_{\perp} \lesssim$

\footnotetext{
${ }^{16}$ Note also that at the $\operatorname{LHC}(\sqrt{s}=8.16 \mathrm{TeV})$ and at mid-rapidity, using (A.5) and (A.7) the range $0.05-0.09 \mathrm{GeV}^{2} / \mathrm{fm}$ for $\hat{q}_{0}$ corresponds to a saturation scale $Q_{s} \equiv \ell_{\perp \mathrm{A}}=1.3-1.8 \mathrm{GeV}$ in a lead nucleus.

${ }^{17}$ We neglect the running of $\alpha_{s}$ at the semi-hard scale $\hat{q} L \sim \mathcal{O}\left(1 \mathrm{GeV}^{2}\right)$.
} 
$\mathcal{O}\left(\ell_{\perp \mathrm{A}}\right)$ could be addressed, as in the case of heavy quarkonium production through $g g \rightarrow$ $G$ [41]. However, the FCEL spectrum associated to $2 \rightarrow 1$ processes has so far been defined and derived assuming the scale $M_{\perp} \equiv\left(M^{2}+p_{\perp}^{2}\right)^{\frac{1}{2}}$ to be hard, $M_{\perp} \gg \ell_{\perp \mathrm{A}}[29,30,39,41]$. Because of this technical limitation, we do not attempt to address FCEL effects on $2 \rightarrow 1$ processes (relevant in the range $1 \mathrm{GeV} \leq p_{\perp} \lesssim \mathcal{O}\left(\ell_{\perp \mathrm{A}}\right)$ ) in light hadron production.

\subsection{Results in $\mathrm{pPb}$ collisions at the $\mathrm{LHC}$}

Calculations of light hadron suppression in $\mathrm{pPb}$ collisions at current top LHC energy, $\sqrt{s}=$ $8.16 \mathrm{TeV}$, are shown here as a function of transverse momentum and rapidity. Although somewhat academic, it is instructive to first discuss the nuclear production ratio for a final gluon pair in a given color state $\left(R_{\mathrm{pA}}^{\mathrm{R}}\right.$ given by $\left.(3.14)\right)$, and then obtain the 'inclusive' hadron suppression through the average (3.13) over color states.

In figure 2 (left) we show the rapidity dependence of $R_{\mathrm{pA}}^{\mathrm{R}}$ at fixed $p_{\perp}=2 \mathrm{GeV}$, for the three color states $\mathrm{R}=\mathbf{1}, \mathbf{8}, \mathbf{2 7}$. (At leading-order, the probability for the $g g$ pair to be in the decuplet state $\mathrm{R}=\mathbf{1 0} \oplus \overline{\mathbf{1 0}}$ vanishes, see appendix B.1.) When the $g g$ final state is color singlet, the fully coherent induced gluon spectrum vanishes, hence no FCEL effect is expected in this case, $R_{\mathrm{pA}}^{1}=1$. More interesting is the suppression of the octet $g g$ final state, $R_{\mathrm{pA}}^{8}<1$, which is predicted in the entire rapidity range considered here, $-6<y<6$. The shape is reminiscent of the suppression predicted for quarkonium in ref. [41]. At $y=0$, the nuclear production ratio is $R_{\mathrm{pA}}^{8} \simeq 0.9$, while the suppression is stronger at larger rapidity, $R_{\mathrm{pA}}^{8} \simeq 0.6$ at $y=6$, due to the pp cross section steeply falling at large $y$. Note that the steeply rising pp cross section at very backward rapidity leads to a slight enhancement $R_{\mathrm{pA}}^{8}>1$ below $y \simeq-6$. For the 27-plet $g g$ final state, the suppression expected from FCEL follows the same pattern, but is more pronounced than for the octet state, due to the larger Casimir, $C_{27}=2\left(N_{c}+1\right)$, in the prefactor (A.2) of the induced gluon spectrum. Shown as a dashed line is the color-averaged nuclear production ratio (obtained from (3.13) by using the probabilities (B.11)), ${ }^{18}$ which we now discuss in more detail.

The light hadron suppression is shown in figure 2 (right) as a function of $y$. The shape of $R_{\mathrm{pA}}^{h}$ at $p_{\perp}=2 \mathrm{GeV}$ is discussed above. Because of the specific dependence of the induced spectrum in $K_{\perp}=p_{\perp} / z$ (recall that in the PDA we must replace $M_{\perp} \rightarrow M_{\bar{\xi}}=$ $K_{\perp} / \sqrt{\bar{\xi}(1-\bar{\xi})}$ in (A.1)-(A.4), see section 3.2), FCEL effects weaken at larger $p_{\perp}$, as can be seen when comparing the predictions at $p_{\perp}=2 \mathrm{GeV}$ and $p_{\perp}=6 \mathrm{GeV}$. In the latter case, the shape is similar and the suppression is more moderate, except at very large rapidity, $y \gtrsim 5$, where the effects of the slope of the cross section are larger at higher $p_{\perp}$ due to the more restricted phase space. Similarly, the enhancement already mentioned at very backward rapidity is now clearly visible below $y \simeq-5$. The $p_{\perp}$ dependence of the nuclear production ratio shown in figure 3 can be simply understood, as $R_{\mathrm{pA}}^{h}$ approaches unity at large $p_{\perp}$ due to the scale dependence of the induced gluon spectrum. At $y=5$ however, the suppression appears to flatten for $p_{\perp}>5 \mathrm{GeV}$, the effect of the scale dependence being compensated by

\footnotetext{
${ }^{18} \mathrm{It}$ turns out to be numerically very close to $R_{\mathrm{pA}}^{8}$, see figure 2 (left). An even more striking coincidence appears in the case of the $q g \rightarrow q g$ underlying process, see section 5 , between the color-averaged modification factor and the color state $\mathrm{R}=\overline{\mathbf{6}}$ of the final $q g$ pair, see figure 6 (left).
} 

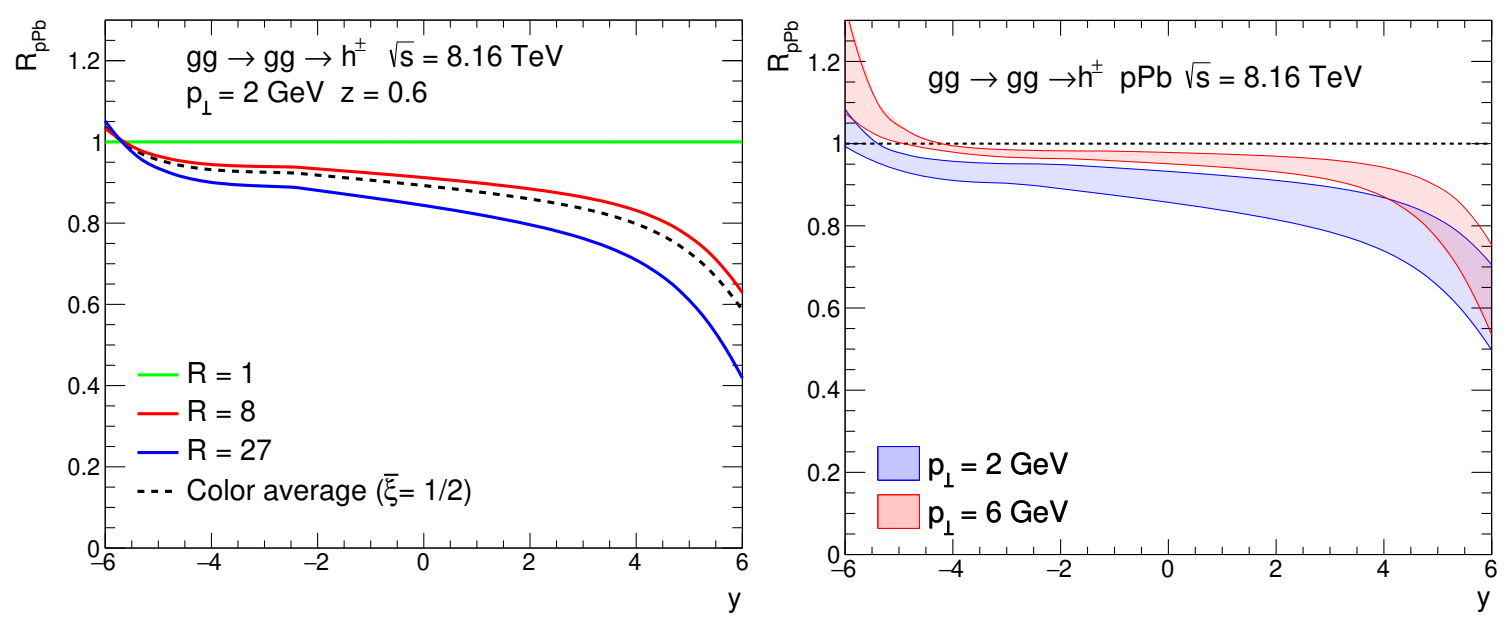

Figure 2. Left: rapidity dependence of $R_{\mathrm{pA}}^{\mathrm{R}}$, eq. (3.14), at $p_{\perp}=2 \mathrm{GeV}$, in the $g g \rightarrow(g g)_{\mathrm{R}}$ channel for $\mathrm{R}=\mathbf{1}$ (green line), $\mathrm{R}=\mathbf{8}$ (red line), and $\mathrm{R}=\mathbf{2 7}$ (blue line). The color-averaged nuclear modification factor $R_{\mathrm{pA}}^{h}$, eq. (3.13), is shown for $\bar{\xi}=1 / 2$ (dashed black line). Right: $R_{\mathrm{pA}}^{h}$ as a function of $y$ and associated uncertainty band, for different values of $p_{\perp}$. Calculations are done at $\sqrt{s}=8.16 \mathrm{TeV}$ in the $g g \rightarrow g g$ channel.

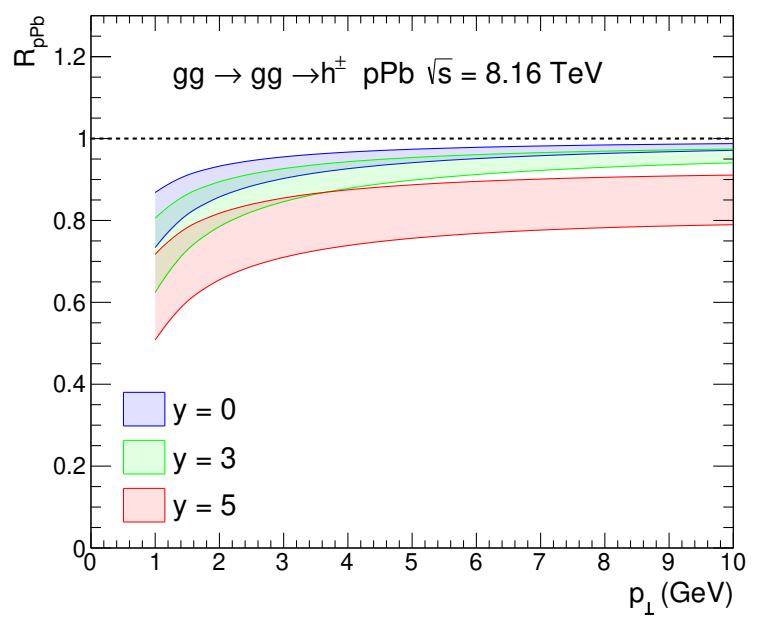

Figure 3. Nuclear modification factor (3.13) as a function of $p_{\perp}$ for different values of $y$. Calculations are done at $\sqrt{s}=8.16 \mathrm{TeV}$ in the $g g \rightarrow g g$ channel.

the strong phase space restriction when the rapidity and transverse momentum are both large.

We now discuss the theoretical uncertainties. As discussed in section 4.2, the uncertainty of our predictions comes from the independent variations of $n, \bar{\xi}, z$ and $\hat{q}_{0}$, resulting in the bands shown in figure 2 (right) and figure 3. In order to give a feeling on the contributions from each parameter variation to the total uncertainty, the curves for $R_{\mathrm{pA}}\left(S_{k}^{+}\right)$ (dash-dotted) and $R_{\mathrm{pA}}\left(S_{k}^{-}\right)$(dashed) are shown individually in figure 4 (left), as a function of rapidity at fixed $p_{\perp}=2 \mathrm{GeV}$. In most of the rapidity range, the upper uncertainty on $R_{\mathrm{pA}}^{h}$ from the (lower) variation of $z, \bar{\xi}$ and $\hat{q}_{0}$ has a similar magnitude, while the variation of $n$ only contributes to the total uncertainty at very forward/backward rapidities, 

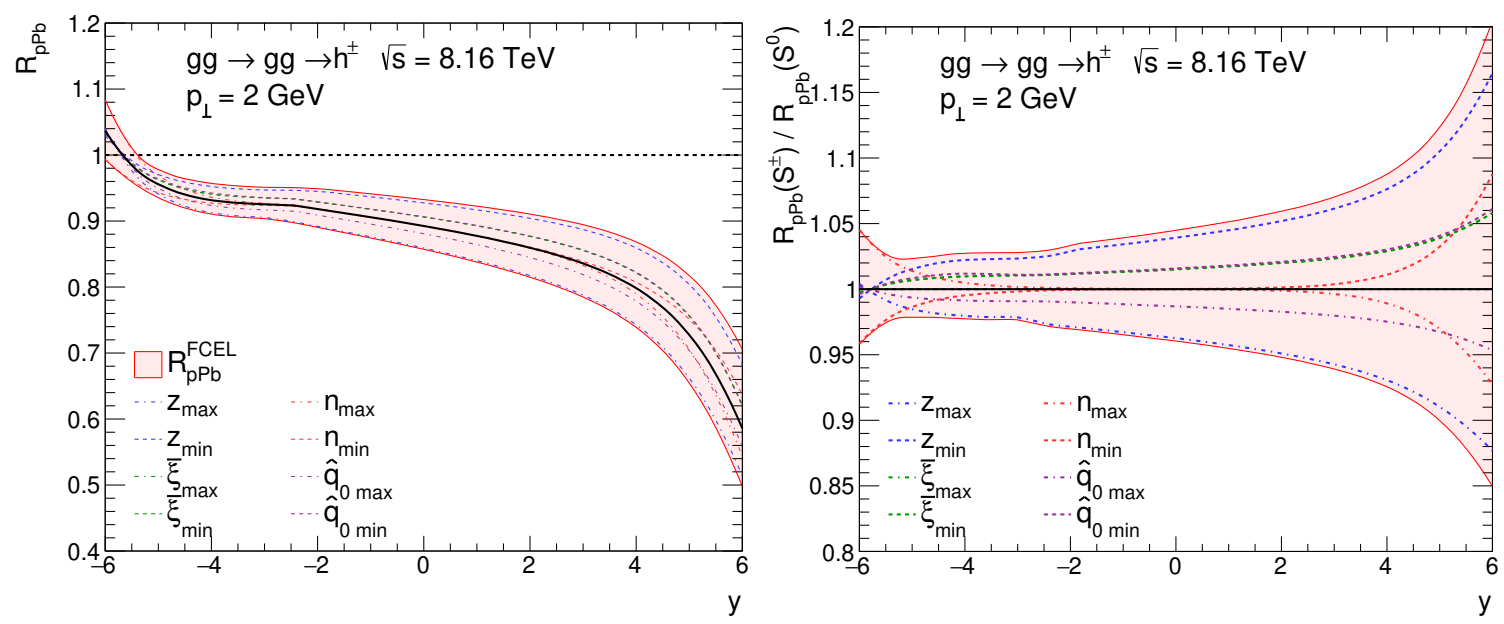

Figure 4. Left: individual contributions $R_{\mathrm{pA}}\left(S_{k}^{ \pm}\right)$to the uncertainty of $R_{\mathrm{pA}}$ at $p_{\perp}=2 \mathrm{GeV}$, in the $g g \rightarrow g g$ channel. Calculations are shown for maximal (dash-dotted lines) and minimal (dashed lines) values of $z$ (blue), $\bar{\xi}$ (green), $n$ (red), and $\hat{q}_{0}$ (purple). The filled band corresponds to the total uncertainty defined by (4.1). Right: relative uncertainty $R_{\mathrm{pA}}\left(S_{k}^{ \pm}\right) / R_{\mathrm{pA}}\left(S^{0}\right)$.

$|y| \gtrsim 5$. The lower uncertainty of $R_{\mathrm{pA}}^{h}$ is dominated by the (upper) variation of $z$, except again at large $|y|$ where the influence of $n$ becomes the largest. This can also be seen in figure 4 (right) showing the individual ratios $R_{\mathrm{pA}}\left(S_{k}^{ \pm}\right) / R_{\mathrm{pA}}\left(S^{0}\right)$ quantifying the relative uncertainty. Remarkably, the uncertainty remains very small, at the level of a few percent around mid-rapidity (and at most $15 \%$ at the largest rapidity considered, $y=6$ ).

We stress that the smallness of FCEL relative uncertainties is expected within our approach, since FCEL is fully determined within perturbative QCD. Moreover, the parameters $\bar{\xi}, z$, and $\hat{q}_{0}$ enter the induced gluon spectrum (A.1) only through the product $\hat{q}_{0} z^{2} \bar{\xi}(1-\bar{\xi})$ in the argument of a logarithm. This logarithm turns out to be large, consistently with the accuracy (3.5) of our approach. Varying the parameters by about $\pm 50 \%$ therefore leads to variations which are formally beyond the leading logarithm, resulting in a narrow uncertainty band. As for the parameter $n$, its variation affects negligibly the predictions, except at very large $|y|$ where it dominates the total relative uncertainty (see figure 4), which however remains moderate.

\subsection{Comparison to data}

Let us now compare the FCEL expectations to the measurements of light hadron suppression in $\mathrm{pPb}$ collisions at $\sqrt{s}=5.02 \mathrm{TeV}$ by the ALICE experiment $[79,80]$.

The measured nuclear production ratio is shown in figure 5 for $\pi^{ \pm}$(top left), $\pi^{0}$ (top right), $K^{ \pm}$(bottom left), and $p / \bar{p}$ (bottom right) production, as a function of $p_{\perp}$. In each panel of figure 5 , we superimpose our prediction for the nuclear production ratio expected from the sole FCEL effect. ${ }^{19}$ Let us note that in our approach, the suppression of different (light) hadron species is expected to be similar. Indeed, although some differences could arise from a significantly different shape of the pp cross section or a different fragmentation

\footnotetext{
${ }^{19}$ This prediction is shown for $p_{\perp} \geq 1 \mathrm{GeV}$ to ensure a perturbative picture $\left(K_{\perp}=p_{\perp} / z \gg \Lambda_{\mathrm{QCD}}\right)$.
} 

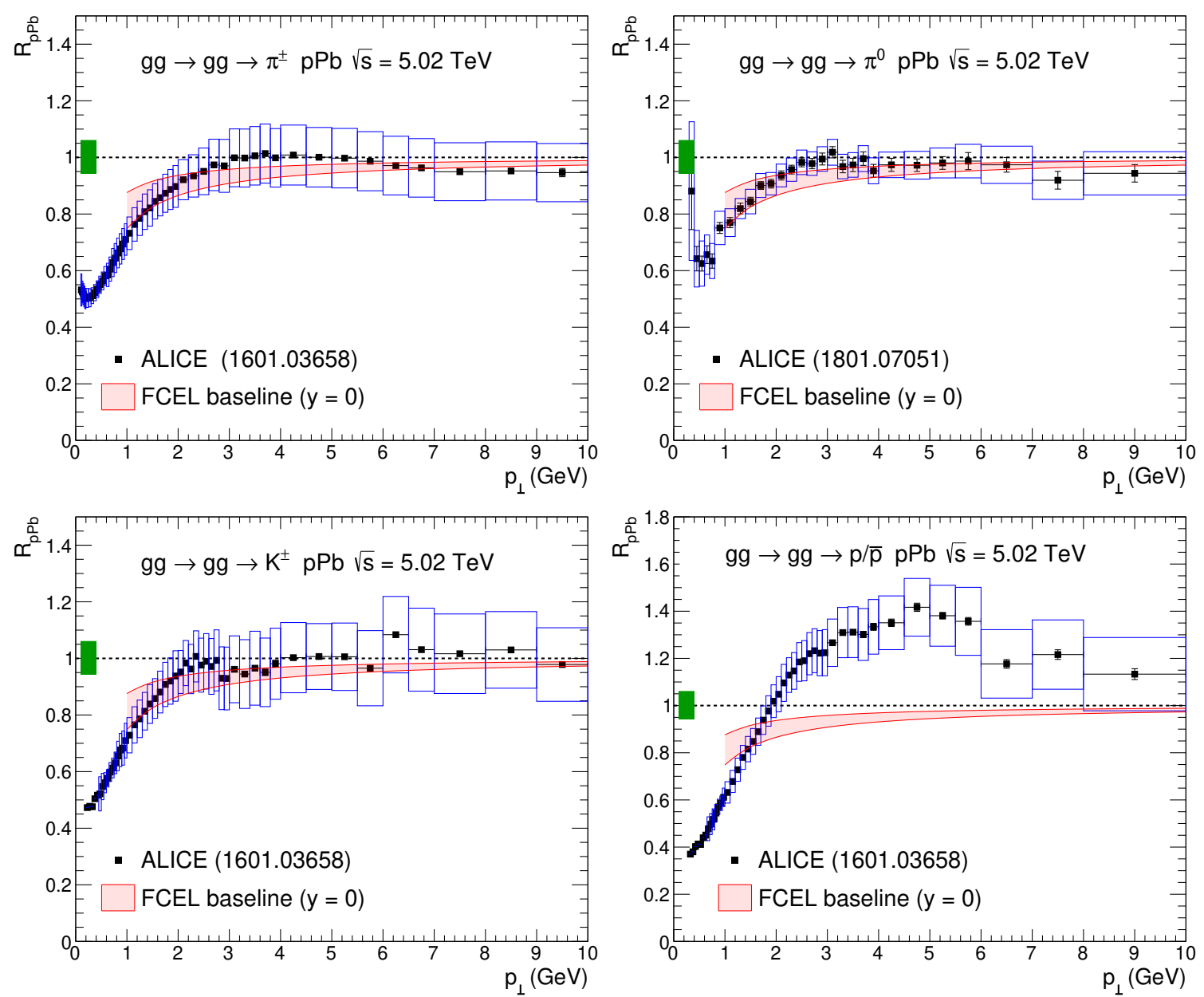

Figure 5. Nuclear modification factor (3.13) in $\mathrm{pPb}$ collisions at $\sqrt{s}=5.02 \mathrm{TeV}$ (red band) in comparison to ALICE charged pion data (top left) [79], neutral pion data (top right) [80], charged kaon data (bottom left) [79], and proton/antiproton data (bottom right) [79]. The baseline calculation assuming FCEL effects only is done in the $g g \rightarrow g g$ channel and at $y=0$.

pattern, such differences should lie within the theoretical uncertainty band which includes the uncertainties associated with the variation of $n$ and $z$.

This baseline prediction - assuming only FCEL effects — proves to be in good agreement with ALICE measurements of pions and kaons. ${ }^{20}$ In particular, the data sometimes attributed to saturation [10, 23-25], nPDF effects [7], and Cronin effect with initial-state energy loss [28], are well reproduced here by fully coherent energy loss alone. Since the uncertainties of the FCEL baseline prediction are small (as discussed in the end of section 4.3) and significantly smaller than those of the measurements, taking into account FCEL should therefore provide strict constraints on other physical processes. Unlike meson production, FCEL alone is clearly not sufficient to explain proton/antiproton data, which exhibit a significant enhancement reminiscent of the Cronin effect above $p_{\perp} \gtrsim 2 \mathrm{GeV}$.

It would be interesting to compare the present baseline predictions at large rapidity, where FCEL effects become the strongest (see figure 2, right). Such measurements could

${ }^{20} \mathrm{~A}$ similar agreement appears between the 'FCEL baseline' and CMS charged hadron data [72]. 
be performed in the near future by the $\mathrm{LHCb}$ experiment in the rapidity region $2<y<$ 4.5 [81]. Measurements at even larger rapidities by the LHCf experiment, $y>8.8$, should also be sensitive to FCEL, although the $p_{\perp}$ coverage in the present data, $p_{\perp} \lesssim 0.8 \mathrm{GeV}[82]$, is limited and barely perturbative.

\section{Other partonic subprocesses}

The results discussed in section 4 have been obtained assuming that hadron production in pp collisions at LHC is dominated by $g g \rightarrow g g$, which as mentioned in section 4.1 is a sensible assumption at mid-rapidity. Here we present the FCEL baseline predictions obtained assuming $q g \rightarrow q g$ (section 5.1), $g q \rightarrow q g$ (section 5.2) and $g g \rightarrow q \bar{q}$ (section 5.3). As we shall see, these predictions are qualitatively similar to those obtained for $g g \rightarrow g g$.

\section{$5.1 \quad q g \rightarrow q g$}

As for $g g \rightarrow g g$, the light hadron suppression in the $q g \rightarrow q g$ channel is computed from (3.13), but with now the accessible color states of the final $q g$ pair being $\mathrm{R}=\mathbf{3}, \overline{\mathbf{6}}, \mathbf{1 5}$. The corresponding probabilities $\rho_{\mathrm{R}}(\xi)$ are given in (B.19) of appendix B.

The rapidity dependence of $R_{\mathrm{pA}}^{\mathrm{R}}$ is shown in figure 6 (left) at fixed $p_{\perp}=2 \mathrm{GeV}$, for the three color states. The shape of $R_{\mathrm{pA}}^{\overline{6}}$ and $R_{\mathrm{pA}}^{15}$ is similar to that of $R_{\mathrm{pA}}^{8}$ and $R_{\mathrm{pA}}^{27}$ in the $g g \rightarrow g g$ channel, see figure 2 (left). Interestingly, the suppression expected in this channel is less pronounced than in the $g g \rightarrow g g$ channel, mostly due to the large negative contribution $C_{a}-C_{b}=C_{F}-N_{c}$ in the prefactor $F_{c}$ of the induced gluon spectrum, eq. (A.2), compared to $C_{a}-C_{b}=0$ for $g g \rightarrow g g$. At large positive rapidity, the $q g \rightarrow q g$ scattering process dominates inclusive hadron production [71]. Consequently, the resulting rapidity dependence of hadron suppression should be less steep than observed in the $g g \rightarrow g g$ channel only, because of the weighted sum over all partonic subprocesses.

As mentioned after eq. (3.15), when the final $q g$ pair is a color triplet, $\mathrm{R}=\mathbf{3}$, lesser (fully coherent) radiation is expected in $\mathrm{pA}$ with respect to pp collisions, due to the negative color prefactor $F_{c}=2 C_{F}-N_{c}=-1 / N_{c}$ in this case. This induced energy gain leads to $R_{\mathrm{pA}}^{3}>1$, as can be seen in figure 6 (left, green solid line). This enhancement is modest since $\left|F_{c}\right|$ is small, but becomes more pronounced at larger rapidity, due to the steepness of the pp cross section: even a small energy gain shifting the rapidity from $y$ to $y-\delta<y$ in eq. (3.15) can lead to a significant enhancement.

The uncertainty band for the 'inclusive' nuclear production ratio $R_{\mathrm{pA}}^{h}$, obtained using the procedure described in section 4.2, is shown in figure 6 (right) for $p_{\perp}=2 \mathrm{GeV}$ and $p_{\perp}=6 \mathrm{GeV}$. It is qualitatively similar to that in the $g g \rightarrow g g$ channel, see figure 2 (right).

\section{$5.2 \quad g q \rightarrow q g$}

In our study the probabilities $\rho_{\mathrm{R}}(\xi)$ associated to a given $a b \rightarrow c d$ process, viewed in the target rest frame where the incoming parton $b$ is softish, are expressed as a function of the variable $\xi$ defined as the light-cone $p^{+}$-momentum fraction of the final parton $c$ w.r.t. the incoming energetic parton a (see section 3.1 and figure 1 ), i.e., $\xi=p_{c}^{+} / p_{a}^{+}$or $\xi=-\hat{t} / \hat{s}$ in terms of the Mandelstam variables of the $a b \rightarrow c d$ process. When going to a frame where 

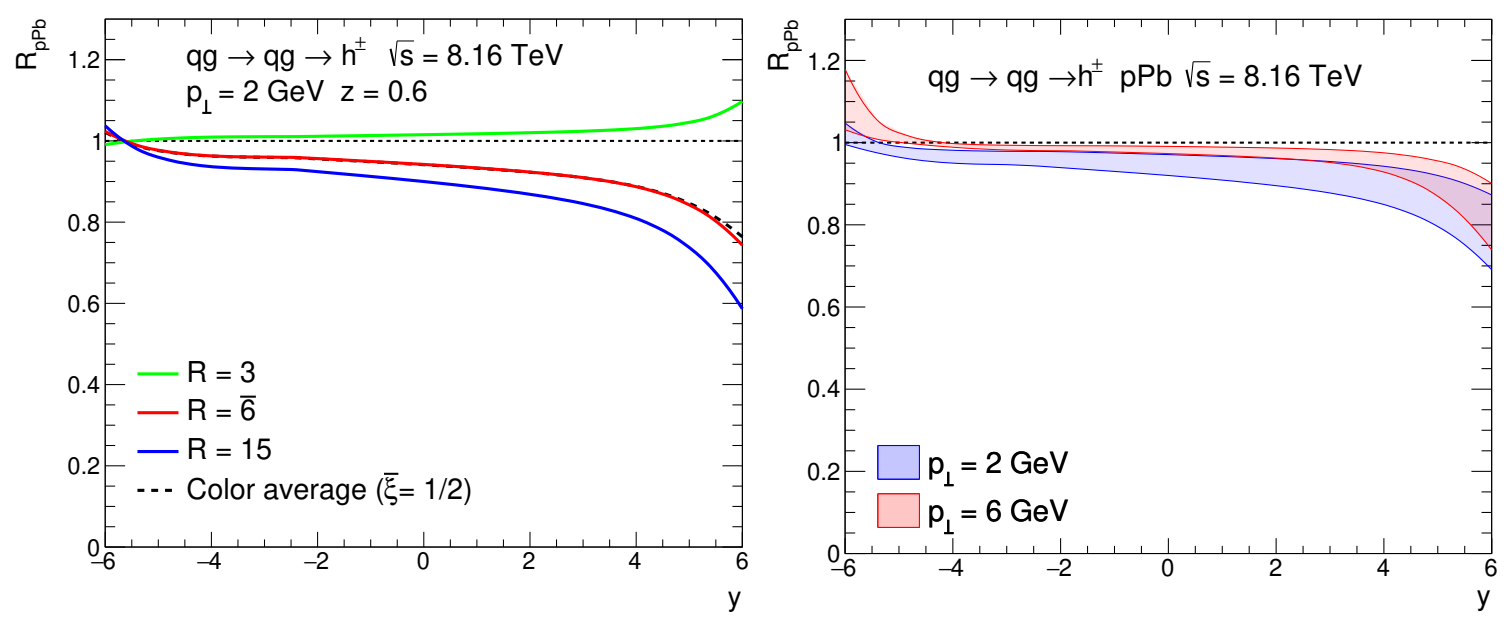

Figure 6. Left: rapidity dependence of $R_{\mathrm{pA}}^{\mathrm{R}}$ at $p_{\perp}=2 \mathrm{GeV}$, in the $q g \rightarrow(q g)_{\mathrm{R}}$ channel for $\mathrm{R}=\mathbf{3}$ (green line), $\mathrm{R}=\overline{\mathbf{6}}$ (red line), $\mathrm{R}=\mathbf{1 5}$ (blue line). The color-averaged nuclear production ratio, $R_{\mathrm{pA}}^{h}$, is shown for $\bar{\xi}=1 / 2$ (dashed black line). Right: rapidity dependence of $R_{\mathrm{pA}}^{h}$ at $p_{\perp}=2 \mathrm{GeV}$ (blue band) and $p_{\perp}=6 \mathrm{GeV}$ (red band) in the $q g \rightarrow q g$ channel.

the incoming parton $a$ is softish (i.e., exchanging $a$ and $b$ in figure 1 and thus considering the $b a \rightarrow c d$ process), the probabilities $\rho_{\mathrm{R}}(\xi)$ are unchanged, but should be expressed (according to the above convention) in terms of the fraction $p_{c}^{+} / p_{b}^{+}=-\hat{u} / \hat{s}=(\hat{s}+\hat{t}) / \hat{s}=1-\xi$. In general, the probabilities associated to the $a b \rightarrow c d$ and $b a \rightarrow c d$ processes are thus related by the $\xi \leftrightarrow 1-\xi$ exchange. Hence, the probabilities associated to the $g q \rightarrow q g$ process directly follow from the probabilities (B.19) associated to $q g \rightarrow q g$.

A more crucial difference between the $g q \rightarrow q g$ and $q g \rightarrow q g$ channels is the sign of $C_{a}-C_{b}$ entering the prefactor $F_{c}$ of the spectrum (see eqs. (A.1)-(A.2)), namely, $C_{a}-C_{b}=$ $N_{c}-C_{F}>0$ in the $g q \rightarrow q g$ channel. Consequently, FCEL effects are significantly larger for $g q \rightarrow q g$ than for $q g \rightarrow q g$, as can be seen by comparing figure 7 with figure 6 . Comparing figure 7 with figure 2, we see that FCEL effects for $g q \rightarrow q g$ are also (slightly) larger than for $g g \rightarrow g g$, the smaller average dijet Casimir $C_{\mathrm{R}}$ in $g q \rightarrow q g$ being overcompensated by a larger $C_{a}-C_{b}$.

The latter observations should be qualified by the fact that at large positive rapidity, the $g q \rightarrow q g$ channel is expected to be significantly less important than $q g \rightarrow q g$ because of the partonic flux in the PDFs, $g\left(x_{1}\right) q\left(x_{2}\right) \ll q\left(x_{1}\right) g\left(x_{2}\right)$ when $x_{1} \gg x_{2}$. For the same reason, the $g q \rightarrow q g$ channel should dominate hadron production only at large negative rapidity $\left(x_{2} \gg x_{1}\right)$, where FCEL effects are actually moderate (except at extreme negative $y$, where nuclear suppression turns into a sizeable nuclear enhancement, $\left.R_{\mathrm{pA}}^{h}>1\right)$.

\section{$5.3 \quad g g \rightarrow q \bar{q}$}

In the $g g \rightarrow q \bar{q}$ channel, the possible $q \bar{q}$ color states are $\mathrm{R}=\mathbf{1}, \mathbf{8}$. The probabilities $\rho_{\mathrm{R}}(\xi)$ to be used in (3.13) are given in eq. (B.22).

The nuclear production ratios $R_{\mathrm{pA}}^{\mathrm{R}}$ for $\mathrm{R}=\mathbf{1 , 8}$ are shown in figure 8 (left). They obviously coincide with those obtained for the same final color states in the $g g \rightarrow g g$ 

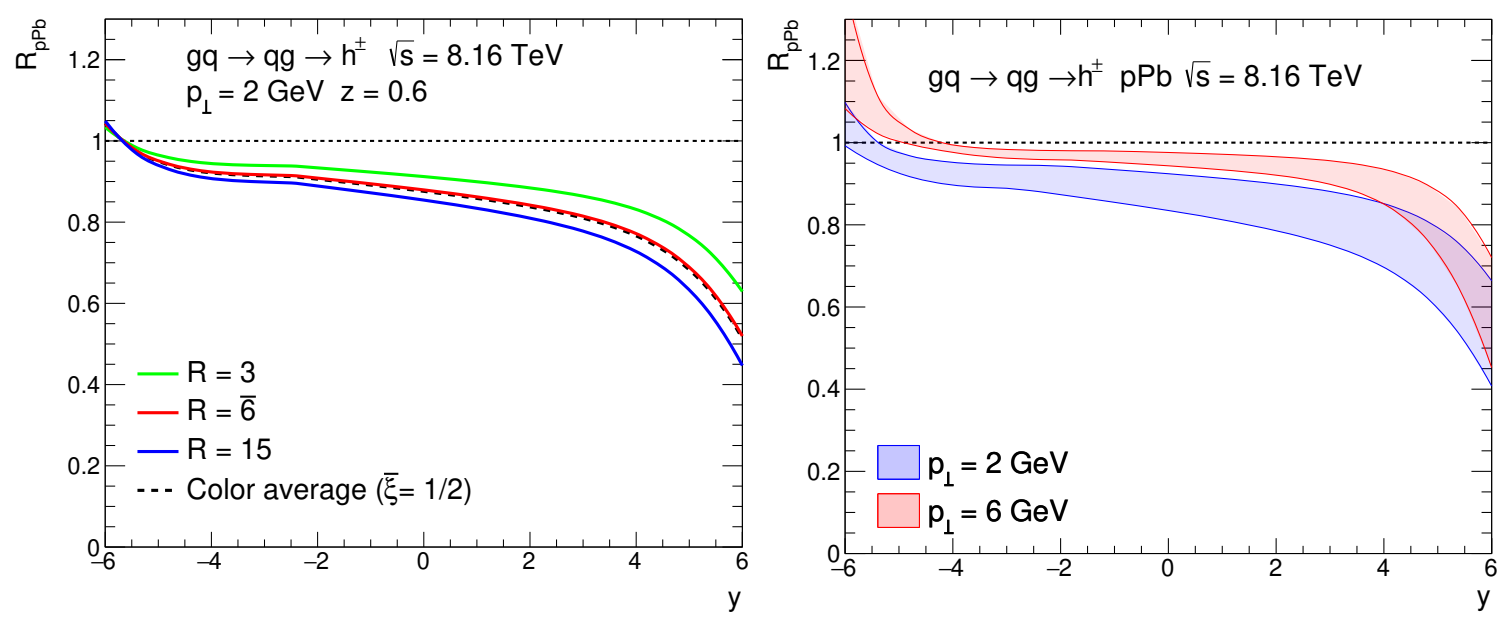

Figure 7. Same as figure 6 in the $g q \rightarrow q g$ channel.
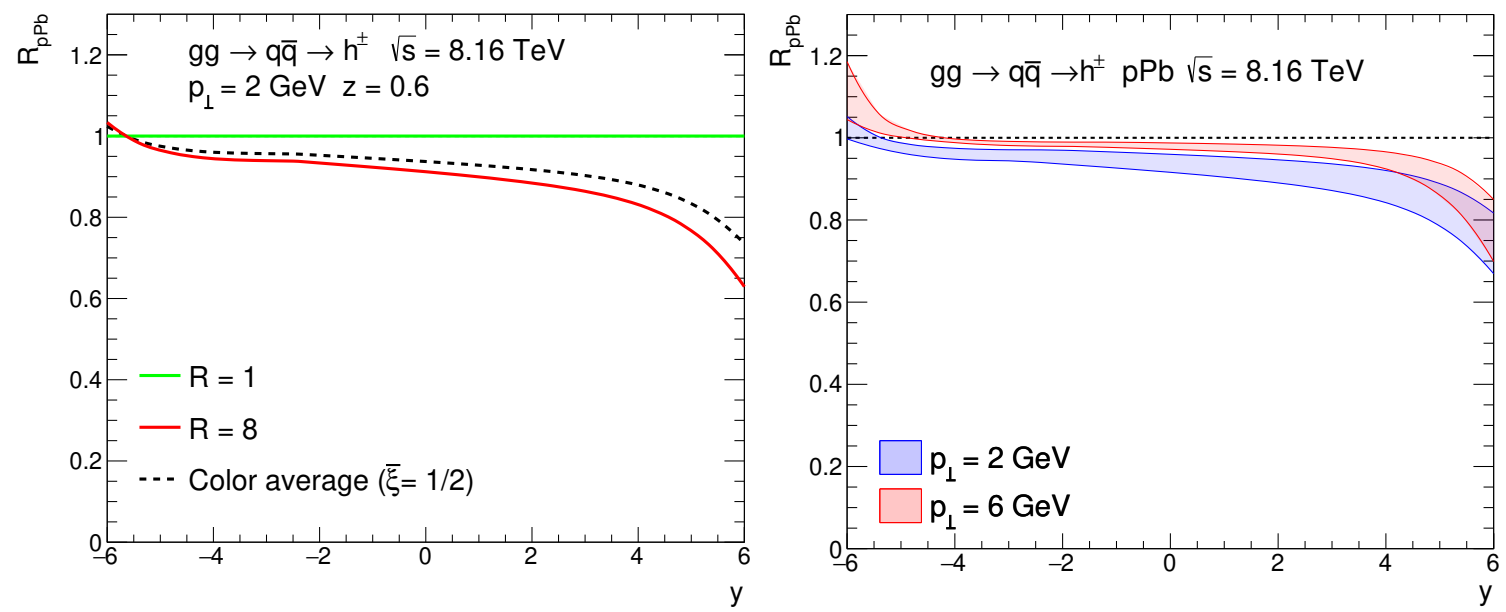

Figure 8. Same as figure 6 in the $g g \rightarrow q \bar{q}$ channel $(\mathrm{R}=\mathbf{1}, \mathbf{8})$.

channel, see figure 2 (left). The difference between the $g g \rightarrow q \bar{q}$ and $g g \rightarrow g g$ channels appears for the color-averaged nuclear production ratio $R_{\mathrm{pA}}^{h}$, see the dashed lines in figure 2 (left) and figure 8 (left), since $\mathrm{R}=\mathbf{2 7}$ is only accessible in the latter channel (and the probabilities $\rho_{\mathrm{R}}(\xi)$ for $\mathrm{R}=\mathbf{1}, \mathbf{8}$ are also different in the two channels). As a consequence, the resulting hadron suppression is slightly less pronounced in the $g g \rightarrow q \bar{q}$ channel.

In figure 8 (right) we show $R_{\mathrm{pA}}^{h}$ as a function of $y$, for $p_{\perp}=2 \mathrm{GeV}$ and $p_{\perp}=6 \mathrm{GeV}$, after taking into account theoretical uncertainties. We see that $R_{\mathrm{pA}}^{h}$ exhibits the same characteristic shape as in the other channels. Surprisingly, the uncertainty band in figure 8 (right) proves narrower than in the other channels, which is due to a numerical coincidence. When $\bar{\xi}$ deviates from its central value $\bar{\xi}=0.5$, both the $q \bar{q}$ invariant mass (3.2) and effective color charge (see figure 9 , right) increase, the former effect leading to lesser suppression, the latter to stronger suppression. It turns out that these two effects almost perfectly balance in the $g g \rightarrow q \bar{q}$ channel, making $R_{\mathrm{pA}}^{h}$ almost independent of $\bar{\xi}$, resulting in a reduced overall theoretical uncertainty. 


\section{Discussion and outlook}

The production of all hadron species in pA collisions is expected to be affected by FCEL [29]. In this work, calculations of the light hadron nuclear production ratio are provided in $\mathrm{pPb}$ collisions at top LHC energy, $\sqrt{s}=8.16 \mathrm{TeV}$, in order to provide FCEL baseline predictions taking only this effect into account. Interestingly, the predictions at $y=0$ (for the $g g \rightarrow g g$ channel) agree well with the $p_{\perp}$ dependence of $R_{\mathrm{pA}}$ of mesons measured by ALICE at $\sqrt{s}=5.02 \mathrm{TeV}$ (while a significant deviation is observed in the case of $p / \bar{p}$ production).

The suppression due to FCEL alone appears to be of the same order of magnitude as nPDF [7] or saturation [10, 23-25] effects at mid-rapidity. FCEL should thus be taken into account when interpreting the pA data, and in particular in nPDF global fit analyses using hadron production data in pA collisions. All the more so as the uncertainties of the FCEL baseline predictions are very small, only $4 \%$ at mid-rapidity (see section 4.3 ), much smaller than the uncertainty of the predictions of $\mathrm{nPDF}$ or saturation effects. As an illustration, we have estimated the nPDF relative uncertainty at mid-rapidity, using nCTEQ15 [3] and EPPS16 [4] nPDF sets, to be $25 \%$ and $40 \%$ at $p_{\perp}=2 \mathrm{GeV}$, respectively. We stress that in our approach, the smallness of FCEL uncertainties is expected from the variation of parameters affecting the induced gluon spectrum beyond the leading logarithm.

The nPDF sets are so far mostly constrained by data which are either insensitive to FCEL (structure functions in DIS, Drell-Yan and W/Z production in pA collisions), or for which FCEL effects are expected to be negligible (large- $p_{\perp}$ jets in $\mathrm{pA}$ collisions). The inclusion of FCEL in nPDF global fit analyses would however affect the treatment of mid-rapidity $\pi^{0}$ measurements in dAu collisions at RHIC which are included in some of these analyses $[3,4]{ }^{21}$ More recently, it has also been proposed to include open-heavy flavour [44, 45] and quarkonium [44] measurements in future global fit analyses, for which the inclusion of FCEL would be necessary for a reliable nPDF extraction.

In order to properly predict FCEL effects away from the mid-rapidity region, one would need to consider other partonic processes eventually overcoming the $g g \rightarrow g g$ process considered in section 4. A first step in this direction has been made in section 5 , where we computed the nuclear production ratio from FCEL effects in the $g g \rightarrow q \bar{q}, q g \rightarrow q g$, and $g q \rightarrow q g$ channels, the last two processes being particularly relevant for hadron production at large forward and large backward rapidity, respectively. Note also that the $g g \rightarrow g g$, $g g \rightarrow q \bar{q}$, and $q g \rightarrow q g$ channels should be the most relevant ones when the nucleus content is dominated by gluons, i.e., at small $x_{2}$, corresponding to the range of mid to large positive hadron rapidities. Since we have found similar results in all the channels considered, our results should provide a conservative uncertainty band for FCEL effects in light hadron nuclear suppression at the LHC.

The $g g \rightarrow q \bar{q}$ channel can be directly generalized to the production of massive quarks, $g g \rightarrow Q \bar{Q}$, relevant to open heavy flavour production in $\mathrm{pPb}$ collisions. The effects of FCEL

\footnotetext{
${ }^{21}$ Note that, before these analyses, EPS08 [83] had used pion production at forward rapidity, which led to nPDFs with a very strong shadowing. But the latter interpretation could obviously overshadow the presence of other, non-universal, nuclear effects increasing with rapidity, such as FCEL. The pion production data at forward rapidity were then discarded in the subsequent nPDF extractions (EPS09 [84], EPPS16 [4]).
} 
in this process, recently measured at forward rapidity by $\mathrm{LHCb}[85,86]$, will be computed in a future study.

In order to eventually include FCEL in nPDF global fit analyses, we envisage computing systematically FCEL effects at all rapidities in a perturbative QCD calculation of hadron production in pA collisions. First, the FCEL calculation presented here should be extended to all possible LO processes (in particular, to $q q \rightarrow q q$ and $q \bar{q} \rightarrow q \bar{q}$ processes). FCEL could then be implemented in a leading-order QCD calculation yielding the relative weights of partonic channels. This program could be also achieved at NLO using FCEL associated to $2 \rightarrow 3$ partonic channels [31] and NLO calculations of hadron production.

\section{Acknowledgments}

This work is funded by "Agence Nationale de la Recherche", grant ANR-COLDLOSS (ANR-18-CE31-0024-02), and by the U.S. Department of Energy, Office of Science, Office of Nuclear Physics under Award Number DE-SC0004286.

\section{A Induced coherent radiation spectrum and quenching weight}

The induced coherent radiation spectrum $\mathrm{d} I / \mathrm{d} \omega$ (together with the associated FCEL quenching weight) is a central quantity in our study. The spectrum associated to $2 \rightarrow 1$ processes was previously derived and discussed in refs. [29, 30, 39, 41]. For a generic $2 \rightarrow 1$ process where the (Casimir) color charges of the partons taken in the incoming proton and in the target nucleus are $C_{a}$ and $C_{b}$, respectively, and the color charge of the outgoing particle is $C_{\mathrm{R}}$, the coherent radiation spectrum is given by ${ }^{22}$

$$
\begin{aligned}
\omega \frac{\mathrm{d} I_{\mathrm{R}}}{\mathrm{d} \omega} & =F_{c} \frac{\alpha_{s}}{\pi}\left\{\ln \left(1+\frac{E^{2} \ell_{\perp \mathrm{A}}^{2}}{\omega^{2} M_{\perp}^{2}}\right)-\ln \left(1+\frac{E^{2} \ell_{\perp \mathrm{p}}^{2}}{\omega^{2} M_{\perp}^{2}}\right)\right\}, \\
F_{c} & =C_{a}+C_{\mathrm{R}}-C_{b} .
\end{aligned}
$$

The induced spectrum (A.1) is defined for a target nucleus A with respect to a target proton $\mathrm{p}$, and thus vanishes when $\mathrm{A}=\mathrm{p}$. In (A.1), $\omega$ and $E$ are the energies of the induced radiation and incoming parton in the target rest frame, $M_{\perp} \equiv\left(M^{2}+p_{\perp}^{2}\right)^{\frac{1}{2}}$ is the transverse mass of the outgoing particle, and $\ell_{\perp \mathrm{A}}$ denotes the transverse momentum broadening of the radiated gluon (as can be inferred from ref. [30]) across a target A.

The associated quenching weight as a function of the energy loss $\varepsilon$ reads [41]

$$
\mathcal{P}_{\mathrm{R}}(\varepsilon, E)=\frac{\mathrm{d} I_{\mathrm{R}}}{\mathrm{d} \varepsilon} \exp \left\{-\int_{\varepsilon}^{\infty} \mathrm{d} \omega \frac{\mathrm{d} I_{\mathrm{R}}}{\mathrm{d} \omega}\right\}=\frac{\partial}{\partial \varepsilon} \exp \left\{-\int_{\varepsilon}^{\infty} \mathrm{d} \omega \frac{\mathrm{d} I_{\mathrm{R}}}{\mathrm{d} \omega}\right\} \equiv \frac{1}{E} \hat{\mathcal{P}}_{\mathrm{R}}\left(\frac{\varepsilon}{E}\right)
$$

\footnotetext{
${ }^{22}$ The parametric dependence of the spectrum (A.1) was first derived in ref. [29] for the scattering of a fast color octet undergoing a single hard gluon exchange in the $t$-channel (in which case $F_{c}=N_{c}+N_{c}-N_{c}=N_{c}$ ). Eq. (A.1), together with the rule (A.2) for the color factor, was shown to hold for any $2 \rightarrow 1$ scattering in ref. [30].
} 
where $\hat{\mathcal{P}}_{\mathrm{R}}$ is a scaling function of the ratio $x \equiv \varepsilon / E$. Using (A.1) this function can be expressed explicitly in terms of the dilogarithm $\operatorname{Li}_{2}(u)=-\int_{0}^{u} \frac{\mathrm{d} v}{v} \ln (1-v)$,

$$
\hat{\mathcal{P}}_{\mathrm{R}}\left(x, \ell_{\perp \mathrm{A}}, M_{\perp}\right)=\frac{\partial}{\partial x} \exp \left\{F_{c} \frac{\alpha_{s}}{2 \pi}\left[\operatorname{Li}_{2}\left(\frac{-\ell_{\perp \mathrm{A}}^{2}}{x^{2} M_{\perp}^{2}}\right)-\mathrm{Li}_{2}\left(\frac{-\ell_{\perp \mathrm{p}}^{2}}{x^{2} M_{\perp}^{2}}\right)\right]\right\}
$$

where the dependence of $\hat{\mathcal{P}}_{\mathrm{R}}$ on $\ell_{\perp \mathrm{A}}$ and $M_{\perp}$ is now emphasized.

The transverse momentum broadening $\ell_{\perp \mathrm{A}}$ is related to the average path length $L_{\mathrm{A}}$ in the target as

$$
\ell_{\perp \mathrm{A}}^{2}=\hat{q} L_{\mathrm{A}}
$$

where $\hat{q}$ is the transport coefficient in cold nuclear matter.

This coefficient is proportional to the gluon distribution $G(x)$ in a target nucleon, as first shown in ref. [32],

$$
\hat{q}=\frac{4 \pi^{2} \alpha_{s}(\hat{q} L) N_{c}}{N_{c}^{2}-1} \rho x G(x, \hat{q} L) \simeq \frac{4 \pi^{2} \alpha_{s} N_{c}}{N_{c}^{2}-1} \rho x G(x),
$$

where $\rho$ is the target nucleon number density, and the scaling violations in the running of $\alpha_{s}$ and in the evolution of the gluon density are neglected since $\hat{q} L \lesssim 1 \mathrm{GeV}^{2}$. The typical value of $x$ at which $x G(x)$ should be evaluated in the r.h.s. of (A.6) is $x=\min \left(x_{0}, x_{2}\right)[32,41]$, where $x_{0}=1 /\left(2 m_{\mathrm{p}} L\right)$ (with $m_{\mathrm{p}}$ the proton mass), and $x_{2}$ is the longitudinal momentum fraction of the target parton (normalized by the nucleus mass number $A$ ) participating to the partonic subprocess as viewed in the p-N c.m. frame. In the present case, $x_{2}=$ $\frac{K_{\perp}}{\sqrt{s}} \frac{e^{-y_{c}}}{1-\xi}=\frac{K_{\perp}}{\sqrt{s}} \frac{e^{-y_{d}}}{\xi}$, where $y_{c}$ and $y_{d}$ are the rapidities of the final partons $c$ and $d$ (see figure 1). Since only the order of magnitude of $x$ matters in $x G(x)$, we have used $x_{2} \sim$ $\left(2 K_{\perp} / \sqrt{s}\right) e^{-y}$, where $y$ is the tagged hadron rapidity. Finally, the $x$-dependence of the gluon distribution can be parametrized by a power-law behavior, $x G(x) \sim x^{\alpha}$, with $\alpha \simeq$ -0.3 , as suggested by small- $x\left(x<10^{-2}\right)$ fits to HERA data [87]. ${ }^{23}$

All together, this leads to the parametrization

$$
\hat{q} \equiv \hat{q}_{0}\left(\frac{10^{-2}}{\tilde{x}_{2}}\right)^{0.3} ; \quad \tilde{x}_{2}=\min \left(x_{0}, x_{2}\right) ; \quad x_{0}=\frac{1}{2 m_{\mathrm{p}} L} ; \quad x_{2} \sim \frac{2 K_{\perp}}{\sqrt{s}} e^{-y},
$$

where the normalization parameter $\hat{q}_{0} \equiv \hat{q}\left(\tilde{x}_{2}=10^{-2}\right)$ can be estimated from various phenomenological studies, see section 4.2 .

\section{B Color state probabilities $\rho_{\mathrm{R}}(\xi)$}

In this appendix we derive the probabilities $\rho_{\mathrm{R}}(\xi)$ for the parton pair produced in a $2 \rightarrow 2$ process to be in color state R. We will consider $g g \rightarrow g g, q g \rightarrow q g$ and $g g \rightarrow q \bar{q}$ processes. The probabilities are calculated in the general case of the $\mathrm{SU}\left(N_{c}\right)$ color group with $N_{c} \geq 3$.

\footnotetext{
${ }^{23}$ We have checked that varying the exponent $\alpha$ by $\pm 10 \%$ around $\alpha=-0.3$ would affect our results by less than $\pm 1 \%$ at $y=0$ and less than $\pm 5 \%$ at $y=6$. These deviations being significantly smaller than those arising from the other parameters (see figure 4), we discarded the uncertainty on $\alpha$ in our study.
} 


\section{B.1 $g g \rightarrow g g$}

The $g g \rightarrow g g$ scattering amplitude can be derived most conveniently using light-cone perturbation theory [88] in light-cone $A^{+}=0$ gauge. It reads (see also [89] and the discussion in [31])

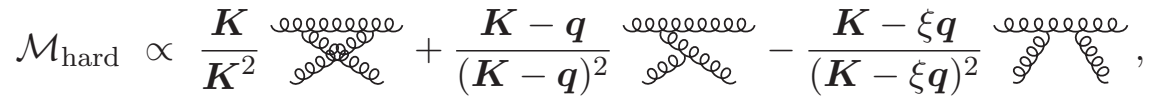

where $\boldsymbol{q}$ is the transverse momentum brought by the gluon from the target nucleus. The graphs in (B.1) represent the color factors associated to each Feynman diagram contributing to the amplitude. ${ }^{24}$ From left to right, the graphs in (B.1) thus read $T^{b} T^{c},\left[T^{c}, T^{b}\right]$ and $T^{c} T^{b}$, where $b$ and $c$ are respectively the color indices of the target gluon and of the final gluon of transverse momentum $\boldsymbol{K}_{c} \equiv \boldsymbol{K}$ and energy fraction $\xi$ (see figure 1), with $T^{a}$ the color generators of the $\mathrm{SU}\left(N_{c}\right)$ adjoint representation. Note that specifying the overall factor in (B.1) is irrelevant for our purpose, since this factor drops out in (B.4).

Using color conservation, ${ }^{25}$

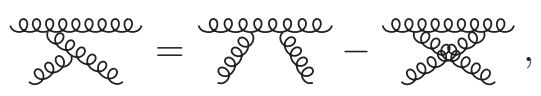

the expression (B.1) can be rewritten as

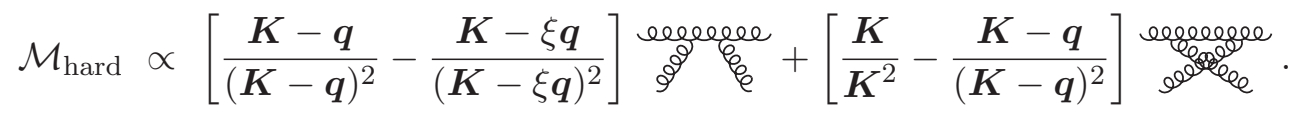

The probability $\rho_{\mathrm{R}}$ for the produced gluon pair to be in color state $\mathrm{R}$ is defined by

$$
\rho_{\mathrm{R}}=\frac{\left|\mathcal{M}_{\text {hard }} \cdot \mathbb{P}_{\mathrm{R}}\right|^{2}}{\left|\mathcal{M}_{\text {hard }}\right|^{2}}
$$

where $\mathbb{P}_{\mathrm{R}}$ is the hermitian projector on the (s-channel) color state $\mathrm{R}$.

We first evaluate the denominator of (B.4). By squaring (B.3) and summing over initial and final color indices we obtain

$$
\left|\mathcal{M}_{\text {hard }}\right|^{2}=C_{\text {in }} K_{\text {in }}\left\{\left(C_{\text {in }}-\frac{N_{c}}{2}\right) \frac{\xi^{2} \boldsymbol{q}^{2}}{\boldsymbol{K}^{2}(\boldsymbol{K}-\xi \boldsymbol{q})^{2}}+\frac{N_{c}}{2} \frac{\boldsymbol{q}^{2}}{(\boldsymbol{K}-\boldsymbol{q})^{2}}\left[\frac{1}{\boldsymbol{K}^{2}}+\frac{(1-\xi)^{2}}{(\boldsymbol{K}-\xi \boldsymbol{q})^{2}}\right]\right\},
$$

where $C_{\text {in }}$ and $K_{\text {in }}$ denote the Casimir and dimension of the incoming (projectile) parton color state, namely, $C_{\text {in }}=N_{c}$ and $K_{\text {in }}=N_{c}^{2}-1$ in the present case.

In order to calculate the numerator of (B.4), which depends on the $g g$ color state R, we recall that for $N_{c}>3$, a $g g$ pair can be in six color representations (see e.g. [92]),

$$
\mathbf{8} \otimes \mathbf{8}=\mathbf{8}_{\mathrm{a}} \oplus \mathbf{1 0} \oplus \mathbf{1} \oplus \mathbf{8}_{\mathrm{s}} \oplus \mathbf{2 7} \oplus \mathbf{0},
$$

where ' 10 ' stands for $\mathbf{1 0} \oplus \overline{\mathbf{1 0}}$, and the representations ordered according to their symmetry properties ( $\mathbf{8}_{\mathrm{a}}$ and $\mathbf{1 0}$ are antisymmetric while $\mathbf{1}, \mathbf{8}_{\mathbf{s}}, \mathbf{2 7}$ and $\mathbf{0}$ are symmetric) are labelled

\footnotetext{
${ }^{24}$ For the pictorial representation of color factors, see for instance refs. [90, 91].

${ }^{25}$ Note that (B.2) is often referred to as the Jacobi identity.
} 
according to their dimensions when $N_{c}=3$. (In particular, $\mathbf{0}$ is a symmetric representation which is absent when $N_{c}=3$.) For $N_{c}>3$ the six color representations $\alpha$ ordered as in the r.h.s. of (B.6) have the following dimensions and Casimirs,

$$
\begin{aligned}
K_{\alpha} & =\left\{N_{c}^{2}-1, \frac{\left(N_{c}^{2}-1\right)\left(N_{c}^{2}-4\right)}{2}, 1, N_{c}^{2}-1, \frac{N_{c}^{2}\left(N_{c}-1\right)\left(N_{c}+3\right)}{4}, \frac{N_{c}^{2}\left(N_{c}+1\right)\left(N_{c}-3\right)}{4}\right\}, \\
C_{\alpha} & =\left\{N_{c}, 2 N_{c}, 0, N_{c}, 2\left(N_{c}+1\right), 2\left(N_{c}-1\right)\right\} .
\end{aligned}
$$

The two color graphs appearing in (B.3) project on the antisymmetric octet, but respectively in the $s$-channel and $u$-channel of the $2 \rightarrow 2$ process,

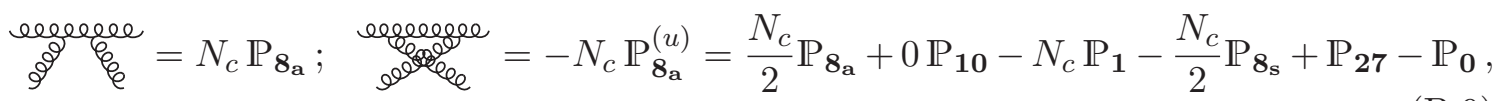

where the $u$-channel projector $\mathbb{P}_{\mathbf{8}_{\mathbf{a}}}^{(u)}$ is written in terms of $s$-channel projectors $\mathbb{P}_{\alpha}[92]$. Inserting (B.9) in (B.3), the numerator of (B.4) (summed over initial and final color indices) is obtained using $\mathbb{P}_{\alpha} \cdot \mathbb{P}_{\beta}^{\dagger}=\mathbb{P}_{\alpha} \cdot \mathbb{P}_{\beta}=\delta_{\alpha \beta} \mathbb{P}_{\alpha}$ and $\operatorname{Tr} \mathbb{P}_{\alpha}=K_{\alpha}$. Dividing by (B.5), and taking finally the limit $|\boldsymbol{q}| \ll|\boldsymbol{K}|$ considered in the present study (see section 3.1), we find the probabilities

$$
\begin{aligned}
& \rho_{\mathbf{8}_{\mathbf{a}}}=\frac{\xi^{2}+(1-\xi)^{2}-1 / 2}{1+\xi^{2}+(1-\xi)^{2}} ; \rho_{\mathbf{1 0}}=0 ; \quad \rho_{\mathbf{8}_{\mathbf{s}}}=\frac{1 / 2}{1+\xi^{2}+(1-\xi)^{2}} ; \\
& \rho_{\mathbf{1}}=\frac{4}{N_{c}^{2}-1} \rho_{\mathbf{8}_{\mathbf{s}}} ; \quad \rho_{\mathbf{2 7}}=\frac{N_{c}+3}{N_{c}+1} \rho_{\mathbf{8}_{\mathbf{s}}} ; \quad \rho_{\mathbf{0}}=\frac{N_{c}-3}{N_{c}-1} \rho_{\mathbf{8}_{\mathbf{s}}} .
\end{aligned}
$$

For $N_{c}=3$, the non-vanishing probabilities are given by

$$
\rho_{\mathbf{2 7}}(\xi)=\frac{3 / 4}{1+\xi^{2}+(1-\xi)^{2}} ; \quad \rho_{\mathbf{1}}(\xi)=\frac{1}{3} \rho_{\mathbf{2 7}}(\xi) ; \quad \rho_{\mathbf{8}}(\xi)=1-\frac{4}{3} \rho_{\mathbf{2 7}}(\xi),
$$

where we have combined the octet representations $\mathbf{8}_{\mathrm{a}}$ and $\mathbf{8}_{\mathrm{s}}$ which have the same dimension and Casimir. The probabilities (B.11) are shown in figure 9 (left).

\section{$\mathrm{B.2} \quad q g \rightarrow q g$}

The probabilities $\rho_{\mathrm{R}}(\xi)$ associated to $q g \rightarrow q g$ can be derived analogously to the $g g \rightarrow$ $g g$ case considered previously. We recall that $\xi$ and $\boldsymbol{K}$ denote the energy fraction and transverse momentum of the final parton denoted as parton $c$ in figure 1, which for a final $q g$ pair we choose to be the gluon.

The relations analogous to (B.1) and (B.2) relevant to $q g \rightarrow q g$ are obtained by replacing the energetic gluon line by a quark line, leading to the analog of (B.3),

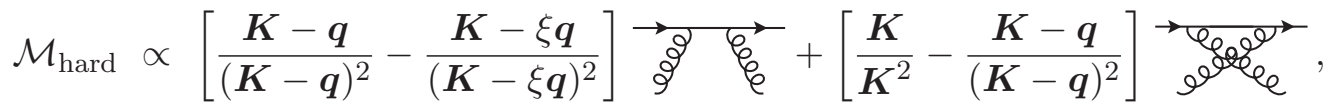

where the graphs now involve the generators of the $\mathrm{SU}\left(N_{c}\right)$ fundamental representation.

It is easy to check that for $q g \rightarrow q g$ the denominator of (B.4) is still given by (B.5), but with now $C_{\text {in }}=C_{F}=\frac{N_{c}^{2}-1}{2 N_{c}}$ and $K_{\text {in }}=N_{c}$. For $N_{c} \geq 3$, the $q g$ pair can be in three different color states (labelled by their dimensions when $N_{c}=3$ ),

$$
\mathbf{3} \otimes \mathbf{8}=\mathbf{3} \oplus \overline{\mathbf{6}} \oplus \mathbf{1 5},
$$



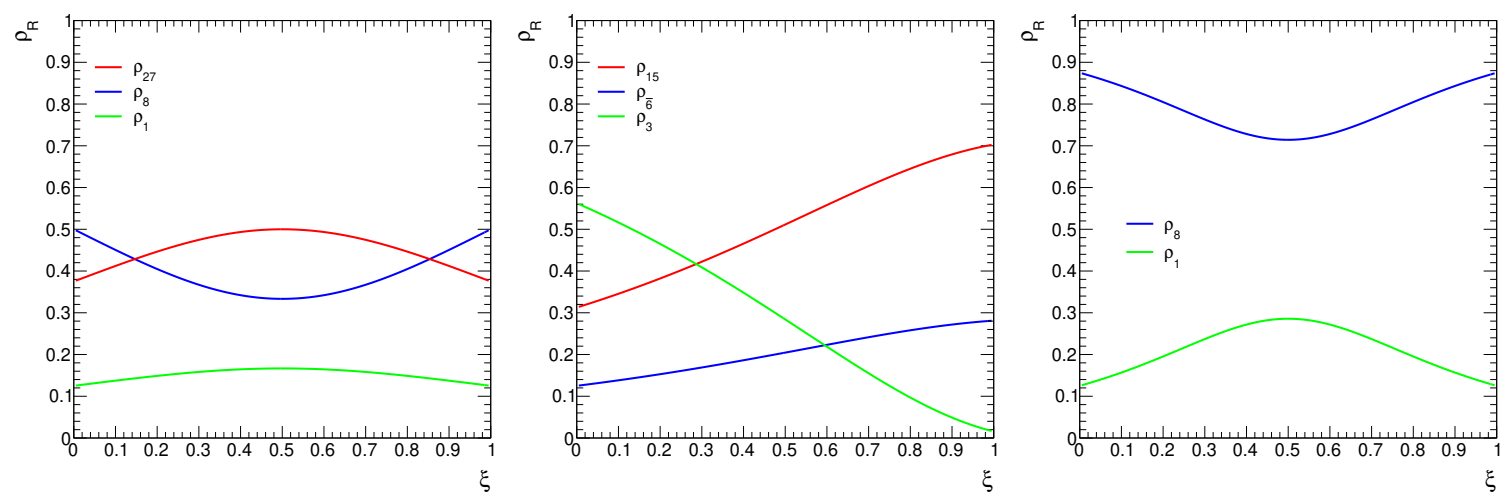

Figure 9. Probabilities $\rho_{\mathrm{R}}(\xi)$ for the parton pair produced in $g g \rightarrow g g$ (left), $q g \rightarrow q g$ (middle), and $g g \rightarrow q \bar{q}$ (right) to be in color state $\mathrm{R}$, as a function of the relative energy fraction $\xi$ carried by parton 1 of the pair (chosen to be the gluon in the $q g \rightarrow q g$ case).

of dimensions and Casimirs

$$
\begin{aligned}
K_{\alpha} & =\left\{N_{c}, \frac{N_{c}\left(N_{c}-2\right)\left(N_{c}+1\right)}{2}, \frac{N_{c}\left(N_{c}+2\right)\left(N_{c}-1\right)}{2}\right\}, \\
C_{\alpha} & =\left\{C_{F}, \frac{\left(N_{c}-1\right)\left(3 N_{c}+1\right)}{2 N_{c}}, \frac{\left(N_{c}+1\right)\left(3 N_{c}-1\right)}{2 N_{c}}\right\},
\end{aligned}
$$

and associated projectors (satisfying the completeness relation $\sum_{R} \mathbb{P}_{R}=\mathbb{1} \equiv \overrightarrow{\text { eеeеeеe }}$ )

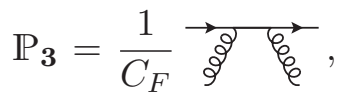

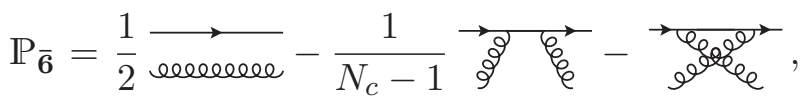

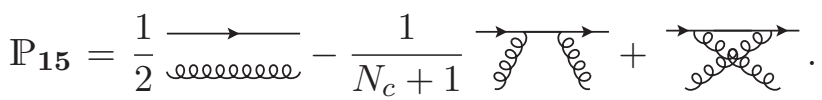

Using (B.16), each color graph of (B.12) can be expressed as a linear combination of projectors, namely,

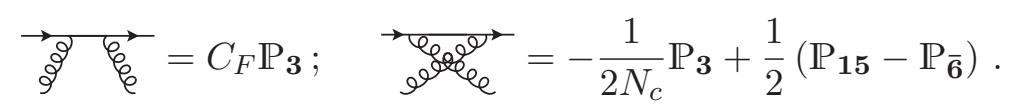

Inserting the latter in (B.12), the numerator of (B.4) is obtained as in the previous section by using $\mathbb{P}_{\alpha} \cdot \mathbb{P}_{\beta}=\delta_{\alpha \beta} \mathbb{P}_{\alpha}$ and $\operatorname{Tr} \mathbb{P}_{\alpha}=K_{\alpha}$. After taking the limit $|\boldsymbol{q}| \ll|\boldsymbol{K}|$, the probabilities associated to $q g \rightarrow q g$ read

$$
\rho_{15}=\frac{\frac{N_{c}\left(N_{c}+2\right)}{4\left(N_{c}+1\right)}}{C_{F} \xi^{2}+N_{c}(1-\xi)} ; \quad \rho_{\overline{\mathbf{6}}}=\frac{\frac{N_{c}\left(N_{c}-2\right)}{4\left(N_{c}-1\right)}}{C_{F} \xi^{2}+N_{c}(1-\xi)} ; \quad \rho_{\mathbf{3}}=\frac{C_{F}\left(\xi-\frac{N_{c}}{2 C_{F}}\right)^{2}}{C_{F} \xi^{2}+N_{c}(1-\xi)} .
$$

For $N_{c}=3$, (B.18) becomes

$$
\rho_{15}(\xi)=\frac{15 / 16}{\frac{4}{3} \xi^{2}+3(1-\xi)} ; \quad \rho_{\overline{\mathbf{6}}}(\xi)=\frac{2}{5} \rho_{15}(\xi) ; \quad \rho_{\mathbf{3}}(\xi)=1-\frac{7}{5} \rho_{15}(\xi) .
$$

Those probabilities are shown in figure 9 (middle). 


\section{B.3 $g g \rightarrow q \bar{q}$}

As in the $q g \rightarrow q g$ case, the calculation of the $g g \rightarrow q \bar{q}$ scattering amplitude in light-cone perturbation theory [88] leads to a result similar to (B.1), up to the appropriate replacement of the color graphs. After using color conservation, we obtain the analog of (B.3) for the $g g \rightarrow q \bar{q}$ case:

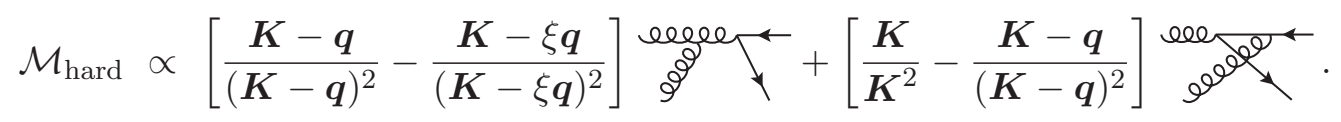

The final $q \bar{q}$ pair can be projected out on either a singlet or an octet $(\mathbf{3} \otimes \overline{\mathbf{3}}=\mathbf{1} \oplus \mathbf{8})$ using the color projectors

$$
\left.\left.\mathbb{P}_{\mathbf{1}}^{q \bar{q}}=\frac{1}{N_{c}}\right\rceil \llbracket ; \quad \mathbb{P}_{\mathbf{8}}^{q \bar{q}}=\longleftarrow-\frac{1}{N_{c}}\right\rceil\lceil.
$$

Using the birdtrack pictorial technique [90, 91], the calculation of the probabilities (B.4) associated to $g g \rightarrow q \bar{q}$ is straightforward and yields (in the limit $|\boldsymbol{q}| \ll|\boldsymbol{K}|$ )

$$
\rho_{1}^{q \bar{q}}(\xi)=\frac{1}{N_{c}^{2}\left(\xi^{2}+(1-\xi)^{2}\right)-1} ; \quad \rho_{\mathbf{8}}^{q \bar{q}}(\xi)=1-\rho_{\mathbf{1}}(\xi) .
$$

The latter probabilities are represented in figure 9 (right) for $N_{c}=3$.

\section{Parametrization of light hadron cross section in pp collisions}

A main input of the model is the double differential light hadron production cross section in pp collisions entering eq. (3.14). Following the same strategy as in earlier papers on quarkonium production [41, 42], the pp production cross section is fitted by a simple analytic form,

$$
\frac{\mathrm{d} \sigma_{\mathrm{pp}}^{\psi}}{2 \pi p_{\perp} \mathrm{d} p_{\perp} \mathrm{d} y} \propto\left(\frac{p_{0}^{2}}{p_{0}^{2}+p_{\perp}^{2}}\right)^{m} \times\left(1-\frac{2 p_{\perp}}{\sqrt{s}} \cosh y\right)^{n}
$$

The parametrization is identical to that used in [42], replacing the quarkonium transverse mass $M_{\perp}$ in eq. (2.11) of [42] by $p_{\perp}$ for light hadron production.

The double differential measurement of the light hadron yields in $\mathrm{pPb}$ collisions at $\sqrt{s}=5.02 \mathrm{TeV}$ has been performed by CMS $[72] .{ }^{26}$ The fits to CMS data, shown in figure 10 for three intervals in $|y|$, lead to the value $n=15 \pm 5$. The values of the other parameters ( $p_{0}$ and $m$ in (C.1)) are irrelevant when computing $R_{\mathrm{pA}}^{h}$ given by eqs. (3.13)-(3.14).

The use of $\mathrm{pPb}$ instead of $\mathrm{pp}$ data in order to parametrize the pp light hadron production rate might seem problematic as these include some nuclear effects. However, those effects have a smooth $y$ and $p_{\perp}$ dependence when compared to the absolute pp cross section, resulting in a relatively flat nuclear modification factor (particularly in the $y$ range of the fitted data, $0.3<|y|<1.8$, see for instance figure 2 , right) thus affecting the cross

\footnotetext{
${ }^{26}$ Other measurements have been performed by ALICE [93] and ATLAS [94] which however lead to looser constraints due to the more restricted $p_{\perp}$ range.
} 

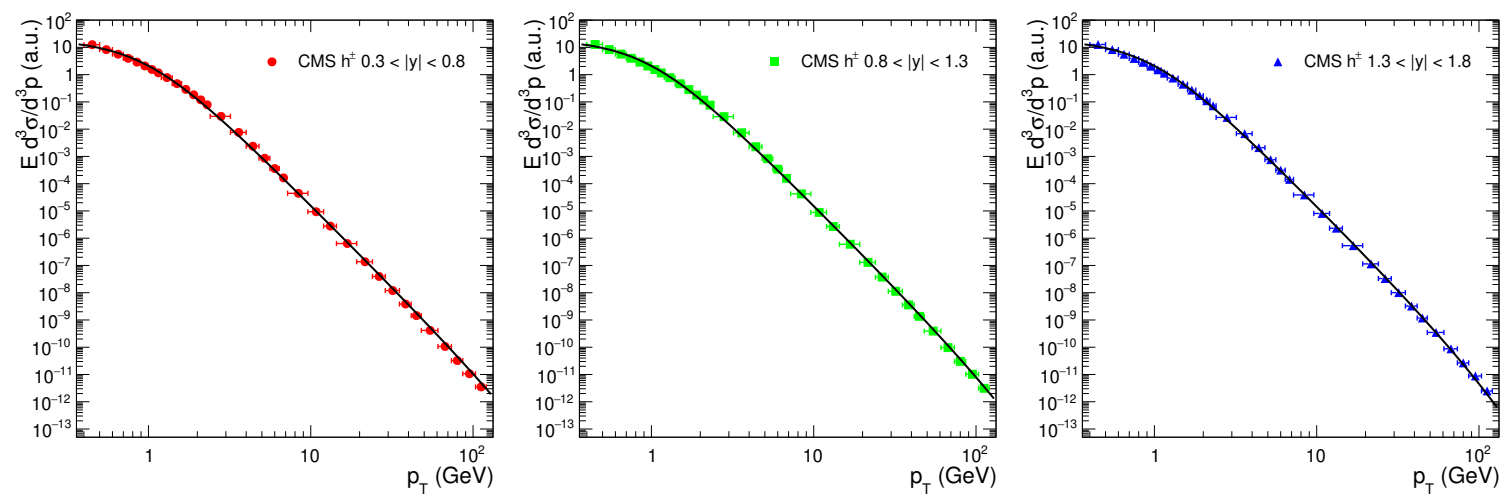

Figure 10. Charged hadron spectra measured by CMS in $\mathrm{pPb}$ collisions at $\sqrt{s}=5.02 \mathrm{TeV}$ in the rapidity ranges $0.3<|y|<0.8$ (left), $0.8<|y|<1.3$ (center), $1.3<|y|<1.8$ (right) [72], compared to the parametrization (C.1).

section normalization but leaving $n$ unchanged. We have checked that inferring the pp cross section from the $\mathrm{pPb}$ data and such a nuclear modification factor, and using the parametrization (C.1), provides a value of $n$ which proves to be fully consistent with the estimate $n=15 \pm 5$. We also remind that the uncertainty of $R_{\mathrm{pA}}^{h}$ associated to the variation of $n$ is subleading with respect to the other sources of uncertainty for rapidities $|y| \lesssim 5$, see figure 4 , right.

Open Access. This article is distributed under the terms of the Creative Commons Attribution License (CC-BY 4.0), which permits any use, distribution and reproduction in any medium, provided the original author(s) and source are credited.

\section{References}

[1] J.C. Collins, D.E. Soper and G.F. Sterman, Factorization of Hard Processes in QCD, in Advanced Series on Directions in High Energy Physics 5, World Scientific (1989), pp. 1-91 [hep-ph/0409313] [INSPIRE].

[2] D. de Florian, R. Sassot, P. Zurita and M. Stratmann, Global Analysis of Nuclear Parton Distributions, Phys. Rev. D 85 (2012) 074028 [arXiv:1112.6324] [INSPIRE].

[3] K. Kovařík et al., nCTEQ15 - Global analysis of nuclear parton distributions with uncertainties in the CTEQ framework, Phys. Rev. D 93 (2016) 085037 [arXiv:1509.00792] [INSPIRE].

[4] K.J. Eskola, P. Paakkinen, H. Paukkunen and C.A. Salgado, EPPS16: Nuclear parton distributions with LHC data, Eur. Phys. J. C 77 (2017) 163 [arXiv:1612.05741] [InSPIRE].

[5] NNPDF collaboration, Nuclear parton distributions from lepton-nucleus scattering and the impact of an electron-ion collider, Eur. Phys. J. C 79 (2019) 471 [arXiv:1904.00018] [INSPIRE].

[6] N. Armesto, Nuclear shadowing, J. Phys. G 32 (2006) R367 [hep-ph/0604108] [INSPIRE]. 
[7] I. Helenius, K.J. Eskola, H. Honkanen and C.A. Salgado, Impact-Parameter Dependent Nuclear Parton Distribution Functions: EPS09s and EKS98s and Their Applications in Nuclear Hard Processes, JHEP 07 (2012) 073 [arXiv: 1205.5359] [INSPIRE].

[8] P. Quiroga-Arias, J.G. Milhano and U.A. Wiedemann, Testing nuclear parton distributions with pA collisions at the TeV scale, Phys. Rev. C 82 (2010) 034903 [arXiv:1002.2537] [INSPIRE].

[9] F. Gelis, E. Iancu, J. Jalilian-Marian and R. Venugopalan, The Color Glass Condensate, Ann. Rev. Nucl. Part. Sci. 60 (2010) 463 [arXiv:1002.0333] [InSPIRE].

[10] J.L. Albacete, A. Dumitru, H. Fujii and Y. Nara, CGC predictions for $p+P b$ collisions at the LHC, Nucl. Phys. A 897 (2013) 1 [arXiv:1209.2001] [InSPIRE].

[11] A. Dumitru, A. Hayashigaki and J. Jalilian-Marian, The Color glass condensate and hadron production in the forward region, Nucl. Phys. A 765 (2006) 464 [hep-ph/0506308] [INSPIRE].

[12] T. Altinoluk and A. Kovner, Particle Production at High Energy and Large Transverse Momentum - 'The Hybrid Formalism' Revisited, Phys. Rev. D 83 (2011) 105004 [arXiv:1102.5327] [INSPIRE].

[13] L.D. McLerran and R. Venugopalan, Computing quark and gluon distribution functions for very large nuclei, Phys. Rev. D 49 (1994) 2233 [hep-ph/9309289] [InSPIRE].

[14] L.D. McLerran and R. Venugopalan, Gluon distribution functions for very large nuclei at small transverse momentum, Phys. Rev. D 49 (1994) 3352 [hep-ph/9311205] [INSPIRE].

[15] J.L. Albacete, N. Armesto, J.G. Milhano, P. Quiroga-Arias and C.A. Salgado, AAMQS: A non-linear QCD analysis of new HERA data at small-x including heavy quarks, Eur. Phys. J. C 71 (2011) 1705 [arXiv: 1012.4408] [INSPIRE].

[16] J. Jalilian-Marian, A. Kovner and H. Weigert, The Wilson renormalization group for low $x$ physics: Gluon evolution at finite parton density, Phys. Rev. D 59 (1998) 014015 [hep-ph/9709432] [INSPIRE].

[17] J. Jalilian-Marian, A. Kovner, A. Leonidov and H. Weigert, The Wilson renormalization group for low x physics: Towards the high density regime, Phys. Rev. D 59 (1998) 014014 [hep-ph/9706377] [INSPIRE].

[18] H. Weigert, Unitarity at small Bjorken x, Nucl. Phys. A 703 (2002) 823 [hep-ph/0004044] [INSPIRE].

[19] E. Iancu, A. Leonidov and L.D. McLerran, The Renormalization group equation for the color glass condensate, Phys. Lett. B 510 (2001) 133 [hep-ph/0102009] [INSPIRE].

[20] E. Iancu, A. Leonidov and L.D. McLerran, Nonlinear gluon evolution in the color glass condensate. 1, Nucl. Phys. A 692 (2001) 583 [hep-ph/0011241] [INSPIRE].

[21] E. Ferreiro, E. Iancu, A. Leonidov and L. McLerran, Nonlinear gluon evolution in the color glass condensate. 2, Nucl. Phys. A 703 (2002) 489 [hep-ph/0109115] [InSPIRE].

[22] J.L. Albacete and C. Marquet, Single Inclusive Hadron Production at RHIC and the LHC from the Color Glass Condensate, Phys. Lett. B 687 (2010) 174 [arXiv:1001.1378] [INSPIRE].

[23] P. Tribedy and R. Venugopalan, QCD saturation at the LHC: Comparisons of models to $p+p$ and $A+A$ data and predictions for $p+P b$ collisions, Phys. Lett. B 710 (2012) 125 [Erratum ibid. 718 (2013) 1154] [arXiv:1112.2445] [INSPIRE]. 
[24] A.H. Rezaeian, $C G C$ predictions for $p+A$ collisions at the $L H C$ and signature of $Q C D$ saturation, Phys. Lett. B $\mathbf{7 1 8}$ (2013) 1058 [arXiv:1210.2385] [INSPIRE].

[25] T. Lappi and H. Mäntysaari, Single inclusive particle production at high energy from HERA data to proton-nucleus collisions, Phys. Rev. D 88 (2013) 114020 [arXiv:1309.6963] [INSPIRE].

[26] B.Z. Kopeliovich, J. Nemchik, I.K. Potashnikova, M.B. Johnson and I. Schmidt, Breakdown of QCD factorization at large Feynman x, Phys. Rev. C 72 (2005) 054606 [hep-ph/0501260] [INSPIRE].

[27] L. Frankfurt and M. Strikman, Energy losses in the black disc regime and correlation effects in the STAR forward pion production in dAu collisions, Phys. Lett. B 645 (2007) 412 [nucl-th/0603049] [INSPIRE].

[28] Z.-B. Kang, I. Vitev and H. Xing, Nuclear modification of high transverse momentum particle production in $p+A$ collisions at RHIC and LHC, Phys. Lett. B 718 (2012) 482 [arXiv: 1209.6030] [INSPIRE].

[29] F. Arleo, S. Peigné and T. Sami, Revisiting scaling properties of medium-induced gluon radiation, Phys. Rev. D 83 (2011) 114036 [arXiv:1006.0818] [INSPIRE].

[30] F. Arleo, R. Kolevatov and S. Peigné, Coherent medium-induced gluon radiation in hard forward $1 \rightarrow 1$ partonic processes, Phys. Rev. D 93 (2016) 014006 [arXiv:1402.1671] [INSPIRE].

[31] S. Peigné and R. Kolevatov, Medium-induced soft gluon radiation in forward dijet production in relativistic proton-nucleus collisions, JHEP 01 (2015) 141 [arXiv:1405.4241] [INSPIRE].

[32] R. Baier, Y.L. Dokshitzer, A.H. Mueller, S. Peigné and D. Schiff, Radiative energy loss and $p_{\perp}$ broadening of high-energy partons in nuclei, Nucl. Phys. B 484 (1997) 265 [hep-ph/9608322] [INSPIRE].

[33] R. Baier, Y.L. Dokshitzer, A.H. Mueller, S. Peigné and D. Schiff, Radiative energy loss of high-energy quarks and gluons in a finite volume quark-gluon plasma, Nucl. Phys. B 483 (1997) 291 [hep-ph/9607355] [INSPIRE].

[34] B.G. Zakharov, Fully quantum treatment of the Landau-Pomeranchuk-Migdal effect in QED and QCD, JETP Lett. 63 (1996) 952 [hep-ph/9607440] [INSPIRE].

[35] B.G. Zakharov, Radiative energy loss of high-energy quarks in finite size nuclear matter and quark-gluon plasma, JETP Lett. 65 (1997) 615 [hep-ph/9704255] [INSPIRE].

[36] N. Armesto, H. Ma, M. Martinez, Y. Mehtar-Tani and C.A. Salgado, Interference between initial and final state radiation in a QCD medium, Phys. Lett. B 717 (2012) 280 [arXiv: 1207.0984] [INSPIRE].

[37] N. Armesto, H. Ma, M. Martinez, Y. Mehtar-Tani and C.A. Salgado, Coherence Phenomena between Initial and Final State Radiation in a Dense QCD Medium, JHEP 12 (2013) 052 [arXiv: 1308.2186] [INSPIRE].

[38] T. Liou and A.H. Mueller, Parton energy loss in high energy hard forward processes in proton-nucleus collisions, Phys. Rev. D 89 (2014) 074026 [arXiv: 1402.1647] [INSPIRE].

[39] S. Munier, S. Peigné and E. Petreska, Medium-induced gluon radiation in hard forward parton scattering in the saturation formalism, Phys. Rev. D 95 (2017) 014014 [arXiv: 1603.01028] [INSPIRE]. 
[40] F. Arleo and S. Peigné, $J / \psi$ suppression in $p-A$ collisions from parton energy loss in cold QCD matter, Phys. Rev. Lett. 109 (2012) 122301 [arXiv:1204.4609] [INSPIRE].

[41] F. Arleo and S. Peigné, Heavy-quarkonium suppression in $p-A$ collisions from parton energy loss in cold QCD matter, JHEP 03 (2013) 122 [arXiv:1212.0434] [INSPIRE].

[42] F. Arleo, R. Kolevatov, S. Peigné and M. Rustamova, Centrality and $p_{\perp}$ dependence of $J / \psi$ suppression in proton-nucleus collisions from parton energy loss, JHEP 05 (2013) 155 [arXiv: 1304.0901] [INSPIRE].

[43] F. Arleo and S. Peigné, Quenching of light hadron spectra in pA collisions from fully coherent energy loss, Phys. Rev. Lett. 125 (2020) 032301 [arXiv:2003.01987] [INSPIRE].

[44] A. Kusina, J.-P. Lansberg, I. Schienbein and H.-S. Shao, Gluon Shadowing in Heavy-Flavor Production at the LHC, Phys. Rev. Lett. 121 (2018) 052004 [arXiv:1712.07024] [InSPIRE].

[45] K.J. Eskola, I. Helenius, P. Paakkinen and H. Paukkunen, A QCD analysis of LHCb $D$-meson data in p+Pb collisions, JHEP 05 (2020) 037 [arXiv: 1906.02512] [INSPIRE].

[46] H. Paukkunen and C.A. Salgado, Constraints for the nuclear parton distributions from $Z$ and $W$ production at the LHC, JHEP 03 (2011) 071 [arXiv:1010.5392] [INSPIRE].

[47] F. Arleo and S. Peigné, Disentangling Shadowing from Coherent Energy Loss using the Drell-Yan Process, Phys. Rev. D 95 (2017) 011502 [arXiv:1512.01794] [inSPIRE].

[48] A. Accardi et al., Electron Ion Collider: The Next QCD Frontier: Understanding the glue that binds us all, Eur. Phys. J. A 52 (2016) 268 [arXiv:1212.1701] [INSPIRE].

[49] ENERGY.GOV, U.S. Department of Energy Selects Brookhaven National Laboratory to Host Major New Nuclear Physics Facility, (2020) https://www.energy.gov/articles/us-departmentenergy-selects-brookhaven-national-laboratory-host-major-new-nuclear-physics.

[50] F. Arleo and S. Peigné, Quarkonium suppression in heavy-ion collisions from coherent energy loss in cold nuclear matter, JHEP 10 (2014) 073 [arXiv:1407.5054] [INSPIRE].

[51] NA3 collaboration, Experimental J/ $\psi$ Hadronic Production from 150 to $280 \mathrm{GeV} / \mathrm{c}, \mathrm{Z}$. Phys. C 20 (1983) 101 [INSPIRE].

[52] S. Katsanevas et al., Nuclear-target effects in $J / \psi$ production in $125 \mathrm{GeV} / \mathrm{c}$ antiproton and $\pi^{-}$interactions, Phys. Rev. Lett. 60 (1988) 2121 [INSPIRE].

[53] FNAL E866/NuSEa collaboration, Measurement of $J / \psi$ and $\psi^{\prime}$ suppression in $p-A$ collisions, Phys. Rev. Lett. 84 (2000) 3256 [nucl-ex/9909007] [INSPIRE].

[54] HERA-B collaboration, Kinematic distributions and nuclear effects of $J / \psi$ production in $920 \mathrm{GeV}$ fixed-target proton-nucleus collisions, Eur. Phys. J. C 60 (2009) 525 [arXiv:0812.0734] [INSPIRE].

[55] NA60 collaboration, $J / \psi$ production in proton-nucleus collisions at 158 and $400 \mathrm{GeV}$, Phys. Lett. B 706 (2012) 263 [arXiv: 1004.5523] [INSPIRE].

[56] A. Andronic et al., Heavy-flavour and quarkonium production in the LHC era: from proton-proton to heavy-ion collisions, Eur. Phys. J. C 76 (2016) 107 [arXiv:1506.03981] [INSPIRE].

[57] PHENIX collaboration, Cold Nuclear Matter Effects on $J / \psi$ Yields as a Function of Rapidity and Nuclear Geometry in Deuteron-Gold Collisions at $\sqrt{s_{\mathrm{NN}}}=200 \mathrm{GeV}$, Phys. Rev. Lett. 107 (2011) 142301 [arXiv:1010.1246] [INSPIRE]. 
[58] PHENIX collaboration, Transverse-Momentum Dependence of the $J / \psi$ Nuclear Modification in $d+$ Au Collisions at $\sqrt{s_{\mathrm{NN}}}=200 \mathrm{GeV}$, Phys. Rev. C 87 (2013) 034904 [arXiv:1204.0777] [INSPIRE].

[59] ALICE collaboration, $J / \psi$ production and nuclear effects in $p-P b$ collisions at $\sqrt{s_{\mathrm{NN}}}=5.02 \mathrm{TeV}$, JHEP 02 (2014) 073 [arXiv: 1308.6726] [INSPIRE].

[60] ALICE collaboration, Rapidity and transverse-momentum dependence of the inclusive $J / \psi$ nuclear modification factor in $p-P b$ collisions at $\sqrt{s_{\mathrm{NN}}}=5.02 \mathrm{TeV}$, JHEP 06 (2015) 055 [arXiv: 1503.07179] [INSPIRE].

[61] LHCb collaboration, Study of $J / \psi$ production and cold nuclear matter effects in $p P b$ collisions at $\sqrt{s_{\mathrm{NN}}}=5 \mathrm{TeV}$, JHEP 02 (2014) 072 [arXiv:1308.6729] [INSPIRE].

[62] F. Cougoulic and S. Peigné, Nuclear $p_{\perp}$-broadening of an energetic parton pair, JHEP 05 (2018) 203 [arXiv: 1712.01953] [INSPIRE].

[63] N. Borghini and F. Gelis, Distribution of multiple scatterings in proton-nucleus collisions at high energy, Phys. Rev. D 74 (2006) 054025 [hep-ph/0607098] [INSPIRE].

[64] N. Borghini and F. Gelis, Are there monojets in high-energy proton-nucleus collisions?, J. Phys. G 34 (2007) S599 [hep-ph/0701140] [INSPIRE].

[65] G.A. Chirilli, B.-W. Xiao and F. Yuan, One-loop Factorization for Inclusive Hadron Production in pA Collisions in the Saturation Formalism, Phys. Rev. Lett. 108 (2012) 122301 [arXiv: 1112.1061] [INSPIRE].

[66] Z.-B. Kang, I. Vitev and H. Xing, Next-to-leading order forward hadron production in the small-x regime: rapidity factorization, Phys. Rev. Lett. 113 (2014) 062002 [arXiv: 1403.5221] [INSPIRE].

[67] B. Ducloué, T. Lappi and Y. Zhu, Single inclusive forward hadron production at next-to-leading order, Phys. Rev. D 93 (2016) 114016 [arXiv:1604.00225] [InSPIRE].

[68] H. Fujii and K. Watanabe, Heavy quark pair production in high energy $p A$ collisions: Quarkonium, Nucl. Phys. A 915 (2013) 1 [arXiv:1304.2221] [InSPIRE].

[69] B. Ducloué, T. Lappi and H. Mäntysaari, Forward $J / \psi$ production in proton-nucleus collisions at high energy, Phys. Rev. D 91 (2015) 114005 [arXiv:1503.02789] [INSPIRE].

[70] Y.-Q. Ma, R. Venugopalan and H.-F. Zhang, $J / \psi$ production and suppression in high energy proton-nucleus collisions, Phys. Rev. D 92 (2015) 071901 [arXiv: 1503.07772] [InSPIRE].

[71] R. Sassot, P. Zurita and M. Stratmann, Inclusive Hadron Production in the CERN-LHC Era, Phys. Rev. D 82 (2010) 074011 [arXiv: 1008.0540] [INSPIRE].

[72] CMS collaboration, Nuclear Effects on the Transverse Momentum Spectra of Charged Particles in $\mathrm{pPb}$ Collisions at $\sqrt{s_{\mathrm{NN}}}=5.02 \mathrm{TeV}$, Eur. Phys. J. C 75 (2015) 237 [arXiv: 1502.05387] [INSPIRE].

[73] CMS collaboration, Evidence for collectivity in pp collisions at the LHC, Phys. Lett. B $\mathbf{7 6 5}$ (2017) 193 [arXiv: 1606.06198] [INSPIRE].

[74] D. d'Enterria, K.J. Eskola, I. Helenius and H. Paukkunen, Confronting current NLO parton fragmentation functions with inclusive charged-particle spectra at hadron colliders, Nucl. Phys. B 883 (2014) 615 [arXiv:1311.1415] [INSPIRE]. 
[75] F. Arleo, C.-J. Naïm and S. Platchkov, Initial-state energy loss in cold QCD matter and the Drell-Yan process, JHEP 01 (2019) 129 [arXiv: 1810.05120] [INSPIRE].

[76] W.K. Brooks and J.A. López, Estimating the Color Lifetime of Energetic Quarks, arXiv:2004.07236 [INSPIRE].

[77] F. Arleo and C.-J. Naïm, Nuclear $p_{\perp}$-broadening of Drell-Yan and quarkonium production from SPS to LHC, arXiv:2004.07188 [INSPIRE].

[78] J. Pumplin et al., Uncertainties of predictions from parton distribution functions. 2. The Hessian method, Phys. Rev. D 65 (2001) 014013 [hep-ph/0101032] [INSPIRE].

[79] ALICE collaboration, Multiplicity dependence of charged pion, kaon, and (anti)proton production at large transverse momentum in $p-P b$ collisions at $\sqrt{s_{\mathrm{NN}}}=5.02$ TeV, Phys. Lett. B 760 (2016) 720 [arXiv:1601.03658] [INSPIRE].

[80] ALICE collaboration, Neutral pion and $\eta$ meson production in $p-P b$ collisions at $\sqrt{s_{\mathrm{NN}}}=5.02$ TeV, Eur. Phys. J. C 78 (2018) 624 [arXiv:1801.07051] [InSPIRE].

[81] LHCb collaboration, Measurement of charged particle multiplicities and densities in pp collisions at $\sqrt{s}=7 \mathrm{TeV}$ in the forward region, Eur. Phys. J. C 74 (2014) 2888 [arXiv: 1402.4430] [INSPIRE].

[82] LHCf collaboration, Measurements of longitudinal and transverse momentum distributions for neutral pions in the forward-rapidity region with the LHCf detector, Phys. Rev. D 94 (2016) 032007 [arXiv: 1507.08764] [INSPIRE].

[83] K.J. Eskola, H. Paukkunen and C.A. Salgado, An Improved global analysis of nuclear parton distribution functions including RHIC data, JHEP 07 (2008) 102 [arXiv:0802.0139] [INSPIRE].

[84] K.J. Eskola, H. Paukkunen and C.A. Salgado, EPS09: A New Generation of NLO and LO Nuclear Parton Distribution Functions, JHEP 04 (2009) 065 [arXiv:0902.4154] [INSPIRE].

[85] LHCb collaboration, Measurement of $B^{+}, B^{0}$ and $\Lambda_{b}^{0}$ production in $p P b$ collisions at $\sqrt{s_{\mathrm{NN}}}=8.16 \mathrm{TeV}$, Phys. Rev. D 99 (2019) 052011 [arXiv: 1902.05599] [INSPIRE].

[86] LHCb collaboration, Study of prompt $D^{0}$ meson production in $p P b$ at $\sqrt{s_{\mathrm{NN}}}=8.16 \mathrm{TeV}$ at LHCb, LHCb-CONF-2019-004 (2019) [CERN-LHCb-CONF-2019-004] [INSPIRE].

[87] K.J. Golec-Biernat and M. Wüsthoff, Saturation effects in deep inelastic scattering at low $Q^{2}$ and its implications on diffraction, Phys. Rev. D 59 (1998) 014017 [hep-ph/9807513] [INSPIRE].

[88] G.P. Lepage and S.J. Brodsky, Exclusive Processes in Perturbative Quantum Chromodynamics, Phys. Rev. D 22 (1980) 2157 [InSPIRE].

[89] J.F. Gunion and G. Bertsch, Hadronization by color bremsstrahlung, Phys. Rev. D 25 (1982) 746 [INSPIRE].

[90] Y.L. Dokshitzer, Perturbative QCD (and beyond), in Lectures on QCD, Lecture Notes in Physics 496, Springer (1997), pp. 87-135 [INSPIRE].

[91] S. Keppeler, Birdtracks for $\mathrm{SU}(N)$, in proceedings of the QCD Master Class 2017, Saint-Jacut-de-la-Mer, France, 18-24 June 2017, Sci. Post. Phys. Lect. Notes 3 (2018) 1 [arXiv: 1707.07280] [INSPIRE]. 
[92] Y.L. Dokshitzer and G. Marchesini, Soft gluons at large angles in hadron collisions, JHEP 01 (2006) 007 [hep-ph/0509078] [INSPIRE].

[93] ALICE collaboration, Transverse momentum spectra and nuclear modification factors of charged particles in pp, $p-P b$ and $P b-P b$ collisions at the LHC, JHEP 11 (2018) 013 [arXiv: 1802.09145] [INSPIRE].

[94] ATLAS collaboration, Transverse momentum, rapidity, and centrality dependence of inclusive charged-particle production in $\sqrt{s_{\mathrm{NN}}}=5.02 \mathrm{TeV} p+P b$ collisions measured by the ATLAS experiment, Phys. Lett. B $\mathbf{7 6 3}$ (2016) 313 [arXiv:1605.06436] [inSPIRE]. 